\title{
Irregular, nanostructured superhydrophobic surfaces: Local wetting and slippage monitored by fluorescence correlation spectroscopy
}

\author{
Xin Zhao, ${ }^{1,2}$ Andreas Best, ${ }^{1}$ Wendong Liu $\odot,{ }^{1}$ Kaloian Koynov $\odot,{ }^{1,}{ }^{*}$ \\ Hans-Jürgen Butt, ${ }^{1}$ and Clarissa Schönecker $\oplus^{1,3, \dagger}$ \\ ${ }^{1}$ Max Planck Institute for Polymer Research, 55128 Mainz, Germany \\ ${ }^{2}$ National Key Laboratory of Vehicular Transmission, Beijing Institute of Technology, \\ 100081 Beijing, People's Rebublic of China \\ ${ }^{3}$ Technical University of Kaiserslautern, 67663 Kaiserslautern, Germany
}

(Received 15 September 2020; accepted 4 May 2021; published 27 May 2021)

\begin{abstract}
Superhydrophobic surfaces used in various applications to enhance flow and reduce drag typically consist of irregular nanostructures. However, many theoretical models and most laboratory microscale experiments dealing with these phenomena are limited to structures consisting of regular microarrays and cannot explain the macroscopic flow enhancement observed in applications. Here, we investigated microscopically the wetting and flow over fluorinated silicon nanofilaments as an example for an application-relevant, irregularly nanostructured, superhydrophobic surface. Using fluorescence correlation spectroscopy with an improved evaluation method, we found that velocity profiles are still nonlinear at distances below $1 \mu \mathrm{m}$ to the surface. Furthermore, we observed that the air layer in between and on the nanofilaments is not continuous on a micrometer length scale. First, there are regions with homogeneous wetting, where the air-water interface regularly touches all uppermost fibers. These regions possess a low slip length $(<5 \mu \mathrm{m})$. Both the wetting and the slip length match with expectations from microarray or homogeneous porous surfaces. Second, there are large patches with air inclusions, which present two orders of magnitude higher slip lengths. Our results contribute to the understanding of the drag reduction observed in applications and can help in designing new, optimized surfaces.
\end{abstract}

DOI: 10.1103/PhysRevFluids.6.054004

\section{INTRODUCTION}

Superhydrophobic or slippery surfaces are investigated and applied for their wide range of particular properties such as fluid-repellency [1-5], antifouling [6-8], anti-icing [9-11], and many more [12-14]. An important property related to all these effects is slippage of the liquid past the surface. Furthermore, slippage reduces drag $[15,16]$ and is therefore interesting for reducing energy costs. Such surfaces are used in nature $[17,18]$ and find technical applications such as coatings for ship hulls [19,20] or components [21,22]. They have been widely investigated theoretically [23-33].

However, while a drag-reducing effect is generally accepted in applications, this is not consistent with the results for simple or model surfaces, which by far cannot reach or explain a similar magnitude of drag reduction. For example, a slippage-induced drag reduction as high as $65 \%$ has been

\footnotetext{
*koynov@mpip-mainz.mpg.de

†schoenecker@mv.uni-kl.de
}

Published by the American Physical Society under the terms of the Creative Commons Attribution 4. 0 International license. Further distribution of this work must maintain attribution to the author(s) and the published article's title, journal citation, and DOI. Open access publication funded by the Max Planck Society. 
reported experimentally [34]. Frequent observations in laminar flow range between $15 \%$ and $30 \%$ [19,34-42]. Such high drag reduction or, correspondingly, slip-length $[38,43]$ values are obtained indirectly by a comparison of macroscopic flow characteristics, such as the flow rate or pressure drop over a plain channel and over one coated with the structured surface. They depend on the geometry of the experimental setup and are related to the slip length of the structured surface, i.e., the larger the slip length, the larger the drag reduction. Since such high drag reduction was only observed macroscopically, but cannot be explained microscopically; it has been critically perceived whether such high drag reduction values could actually be real [44]. Fluid dynamic considerations show that to obtain a reduction in pressure of $50 \%$ in a channel of $500 \mu \mathrm{m}$ in height, a slip length of more than $60 \mu \mathrm{m}$ would be required. If we consider that the presence of a coating also constricts the channel, a $10-\mu \mathrm{m}$-thick coating would increase the necessary slip length to about $80 \mu \mathrm{m}$. Larger channel heights, i.e., also larger-scale applications, would require even more slip. On the other hand, direct measurements that resolve the flow close to a superhydrophobic surface on the scale of the surface structures are rare [45-48] and only report small effective slip lengths, in general up to around $2 \mu \mathrm{m}$. Similar values have been found in other direct measurements $[33,49,50]$. These values are not high enough to reach a drag reduction even close to those claimed in macroscopic experiments.

A similar discrepancy exists with theoretical models. For example, a superhydrophobic surface with $90 \%$ free interface and $10 \mu \mathrm{m}$ grooves theoretically has an effective slip length between only 3 and $6.5 \mu \mathrm{m}$, depending on the flow direction [51]. Smaller structures, which are advantageous with respect to Cassie state stability [52,53] and therefore favored in applications, should possess an even smaller slip length. For nanostructured surfaces with $1 \mu \mathrm{m}$ spacing, the above theory would predict an effective slip length even below $1 \mu \mathrm{m}$. The reason for this is that the slip length scales with the distance between the solid parts of the surface [51]. At these parts, where the liquid is in actual contact with the surface, there is a no-slip boundary condition (or a very small molecular slip at maximum). So, the further the solid protrusions are away from each other, the more the flow can accelerate after the no-slip condition before it has to decelerate again to meet the no-slip condition on the following solid part. Nanostructured surfaces should therefore have a nanoscopic slip length, at maximum a few $\mu \mathrm{m}$.

A significant difference between most surfaces used in macroscopic experiments and those used for local flow studies and theoretical models is their surface geometry. Large-scale applications call for coating methods that can be used for larger areas, like spraying, dipping, or sandblasting, and hence usually lead to irregular patterns of the surface geometry. In contrast, both local flow measurements and theoretical modeling are typically undertaken for defined, regular surface structures. Also, irregular structures are usually much smaller. Reducing size enhances wetting stability and is therefore an important prerequisite for applications. Hence, the local flow phenomena on irregular, nanostructured superhydrophobic surfaces that can presumably lead to a double-digit enhancement of drag [19,34-37,39-42,54,55] are of high interest for applications [22,56-58]. This also involves the question of how the air-water interface actually makes contact with the locally varying boundary [20].

In this paper, we perform a case study on local slip and flow on an application-relevant irregularly nanostructured surface. Using confocal microscopy and fluorescence correlation spectroscopy (FCS) [59-62], we show that such surfaces accommodate large inclusions of air in a stable Cassie state, which have a comparatively large slip length. We also find that the velocity profile perpendicular to the surface may be nonlinear below $1 \mu \mathrm{m}$ close to the surface. Since this is a source of error for slip-length measurements, a resolution of this velocity nonlinearity at the nanoscale is needed for accurate measurements. We modified FCS to be able to measure in this range close to nontransparent surfaces.

\section{EXPERIMENTAL SETUP}

As an example of an irregular, application-relevant superhydrophobic surface, we employed silicone nanofilaments (Fig. 1). They were chemically grown from a solution of toluene and 




(b) fluorescent particle nanofilament surface
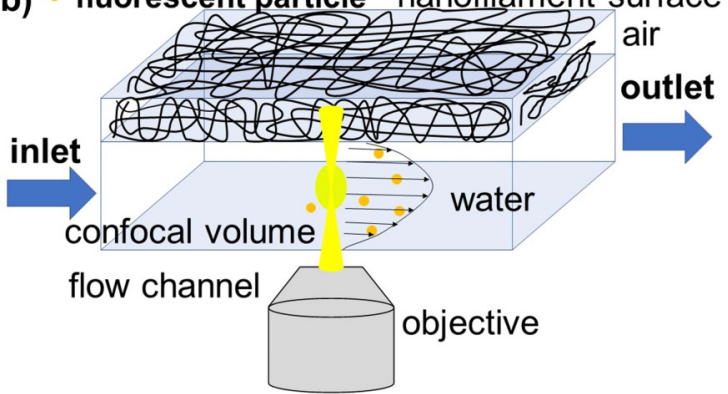

FIG. 1. (a) Scanning electron microscopy image of the nanofilament coating. (b) Schematic of the flow setup.

trichloromethylsilane and are fluorinated $[14,63]$ directly on a glass slide. The fiber geometry acts as an overhang structure that leads to a high energy barrier for an air-water interface to penetrate into the structure and thus stabilizes the Cassie state. The static contact angle of water was measured to be about $163 \pm 3^{\circ}$ and the one of hexadecane $155 \pm 5^{\circ}$, which also characterizes the surface as superamphiphobic. The main property of the surface for our application is, however, the good stability of the Cassie state, since there is no droplet but the surface is completely covered with water. Even under shear flow, no water penetrating into the fiber structure or leaking of the air could be observed. For our experiments, the thickness of the nanofilament layer was 10 to $20 \mu \mathrm{m}$, such that the reflected light from the air-water interface and from the supporting glass slide-nanofilament interface could always be clearly distinguished.

All experiments were performed in a microchannel system $(150 \mu \mathrm{m}$ in height, $2 \mathrm{~mm}$ wide, $20 \mathrm{~mm}$ long). It consists of two glass slides separated by an acrylate tape of defined thickness (Tesa 4972) acting as a spacer. The upper glass slide is the one coated with the silicone nanofilaments, while the lower one is a coverslip [Fig. 1(b)]. The channel is fixed in a metal holder that keeps it securely fixed on an inverted microscope. Purified water was pumped through the channel with a Reynolds number $\approx 3(\operatorname{Re}=\rho V H / \mu$, where $H$ is height of the channel, $V$ the mean velocity, $\rho$ the density and $\mu$ the viscosity.) using an adjustable micropump (mp6, Bartels Mikrotechnik).

Confocal microscopy and FCS experiments were performed on a commercial setup (Carl Zeiss, Jena, Germany) consisting of the modules ConfoCor 2 and LSM 510 coupled to an Axiovert 200 inverted microscope equipped with a C-Apochromat 40x/1.2W water immersion objective. As fluorescent tracers, Qdot $585 \mathrm{ITK}^{\mathrm{TM}}$ carboxyl quantum dots (Invitrogen) with a hydrodynamic radius of $R_{h} \approx 12 \mathrm{~nm}$ and a concentration of about $10^{-9} \mathrm{M}$ were used. $\mathrm{K}_{2} \mathrm{HPO}_{4}$ was added as buffer solution ( $1 \mathrm{mM}$, Debye length, $\lambda_{D} \approx 5 \mathrm{~nm}$ ) to prevent possible electrostatic repulsion from keeping the tracer particles away from the interface [64]. For excitation, the 488-nm line of an Argon laser, fiber-coupled to the microscope, was used.

Before each measurement, the whole microfluidic system was flushed with purified water for 30 minutes to prevent any substances from accumulating at the air-water interface since concentration differences in these substances could potentially drive Marangoni flows. Then, the measurement procedure was as follows: After checking Cassie state stability by confocal imaging in reflection mode, the confocal detection volume was moved stepwise in a direction perpendicular to the surface, while recording FCS autocorrelation curves at each position. The step size varied from $0.2 \mu \mathrm{m}$ very close to the surface up to $2 \mu \mathrm{m}$ further away from the wall. The experimentally recorded autocorrelation curves were then fitted with a theoretical model to obtain the velocity. The standard theoretical model for an FCS autocorrelation curve $G(\tau)$ with flow reads [65]

$$
G(\tau)=\frac{1}{N} \frac{1}{1+\frac{\tau}{\tau_{D}}} \frac{1}{\sqrt{1+\frac{\tau}{\tau_{D} S^{2}}}} e^{-\left(\frac{\tau U}{r_{0}}\right)^{2} \frac{1}{1+\frac{\tau}{\tau_{D}}}} .
$$



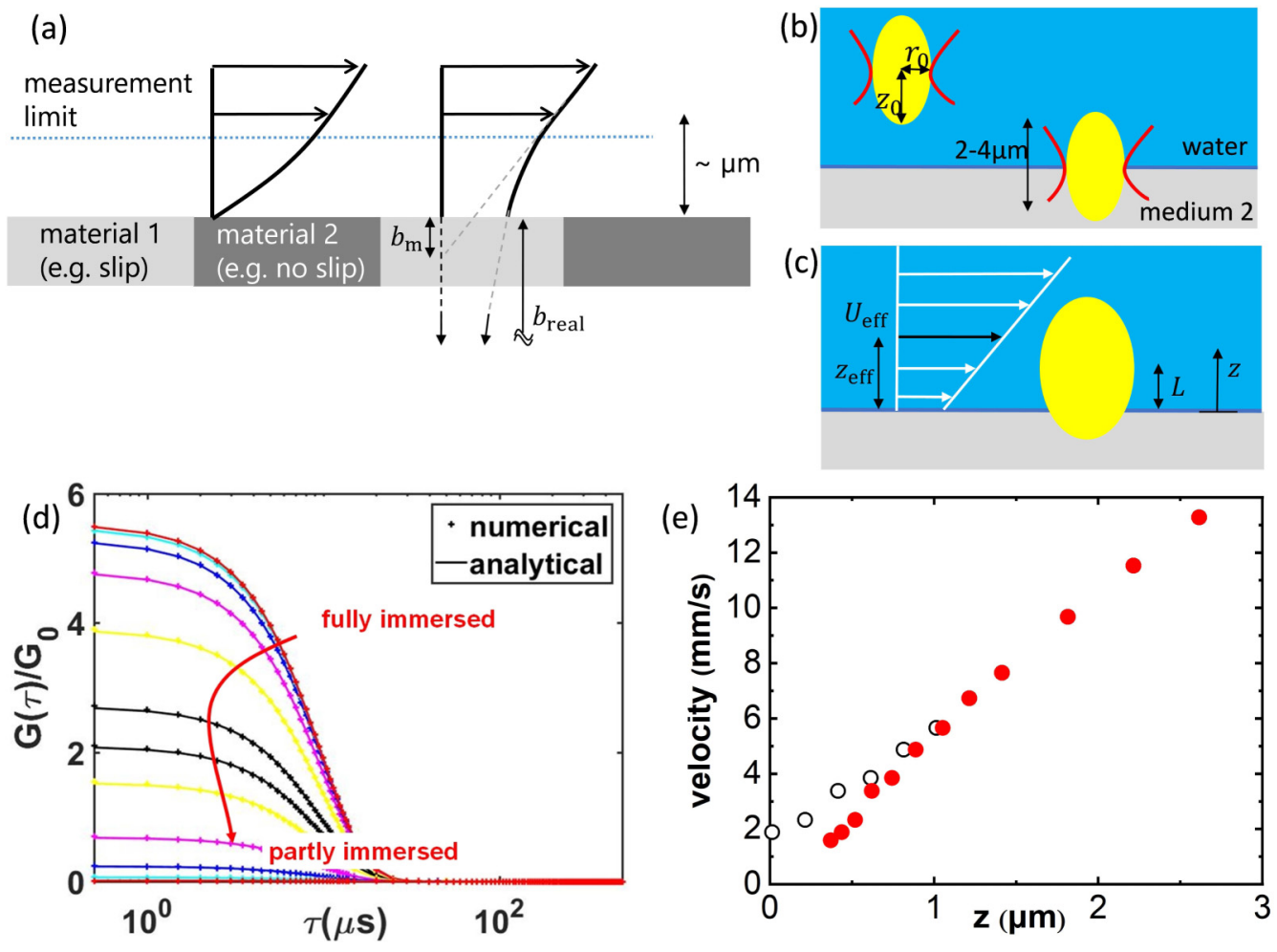

FIG. 2. (a) Schematic of velocity variations due to changing boundary conditions. (b) Schematic of the detection volume. (c) Definition of the parameters for Eq. (2). (d) Comparison of autocorrelation curves calculated by the full numerical solution of the autocorrelation function for a partly immersed detection volume and their fits with Eq. (2). (e) Comparison of velocity profiles recorded with FCS close to a bare glass surface and evaluated with the theory for a fully immersed volume (open circles) and with Eq. (2) (filled circles).

Here, $N$ is the average number of fluorescent particles in the detection volume. The size of the detection volume is characterized by the structure parameter $S=z_{0} / r_{0}$ [Fig. 2(b)]. $\tau_{D}$ is the diffusion time of the fluorescent particles through the detection volume that is directly related to their diffusion coefficient $D=r_{0}^{2} /\left(4 \tau_{D}\right)$. $U$ is the flow velocity at the position of the detection volume.

The standard expression Eq. (1) assumes a detection volume fully immersed in the liquid phase $[65,66]$, which will turn out to be crucial in the following and will lead us to the derivation of an extended theoretical model that is used in the experiments. Finally, the local slip length was obtained by extrapolating the measured velocity profiles below the surface.

\section{FCS EVALUATION METHOD}

For an accurate slip measurement, it is crucial to measure as close to the surface as possible, because, as mentioned, the slip length is based on the extrapolation of the velocity profile below the surface. Undetected changes in the slope of the velocity profile close to the surface would lead to a wrong estimation of the slip length [Fig. 2(a)]. FCS is capable to measure local velocities down to about a distance of $1 \mu \mathrm{m}$ to a surface [47,65]. As described above, FCS velocity measurements are performed by fitting a theoretical model to the autocorrelated fluorescence signal collected from a confocal detection volume as a flow loaded with small fluorescent tracers passes through the volume $[65,66]$. However, all such FCS models are based on a detection volume fully immersed in the liquid phase. Below about $1 \mu \mathrm{m}$ to an interface, the confocal detection volume starts to significantly leave 
the liquid and enter into medium 2. Medium 2 would be the coating or air in our case [Fig. 2(b)]. This leads to artifacts, so the corresponding values are typically discarded. In an attempt to address this issue, we extended the standard FCS technique to account for partly immersed detection volumes.

Furthermore, the standard assumption for FCS velocity measurements is that the velocity is constant throughout the detection volume $[65,66]$. This case is treated in Appendix A. However, for flows very close to surfaces, the velocity gradient may be large. We model this variation as a linear velocity profile within the detection volume and solve the general form of the FCS autocorrelation function, assuming fluorescence is only emitted from within the water phase (see Appendix A). One term that appears during this calculation process can, however, not be solved explicitly in contrast to the fully immersed case. Therefore, we derived an approximate analytical expression for this specific term, which essentially models the solution for the partly immersed case as a fraction of the fully immersed one. A longer calculation leads to the autocorrelation function for a flow with a linear velocity across the detection volume which is placed at a distance $L$ to the interface [Fig. 2(c):

$$
G(\tau, L, U)=N_{2}(L, D) \frac{1}{1+\frac{\tau}{\tau_{D}}} \frac{1}{\sqrt{1+\frac{\tau}{\tau_{D} S^{2}}}} e^{-\left(\frac{\tau U_{\text {eff }}}{r_{0}}\right)^{2} \frac{1}{1+\frac{\tau}{\tau_{D}}}} .
$$

Note that Eq. (2) in principle has the same mathematical form as the standard expression for a constant velocity $U$ through a fully immersed detection volume Eq. (1). However, here $U_{\text {eff }}$ is an effective velocity within the detection volume that is allocated to the position

$$
z_{\text {eff }}=\frac{z_{0} e^{-2 \frac{L^{2}}{z_{0}^{2}}}}{\sqrt{2 \pi}\left(1+\operatorname{erf}\left(L \sqrt{\frac{2}{z_{0}^{2}}}\right)\right.}
$$

such that, despite the varying velocity profile, a single velocity value per measurement can be derived [Fig. 3(c)]. The second difference between the standard and the current case is that the prefactor $N_{2}(L, D)$ does not have the same form and hence meaning as in the fully immersed case. In the fully immersed case, a similar prefactor $1 / N$ reflects the inverse of the average number of particles in the detection volume. For the partially immersed case, $N_{2}(L, D)$ is a general fitting parameter. It converges to $1 / N$ as the detection volume fully immerses into the liquid bulk. With respect to the analytical approximations made during the derivation, the approximated terms in the final expression are the prefactor $N_{2}$, which, regarding the experimental procedure is a fitting parameter anyhow, the square root and $U_{\text {eff }}$ terms in Eq. (2), and the expression for $z_{\text {eff }}$.

(a)

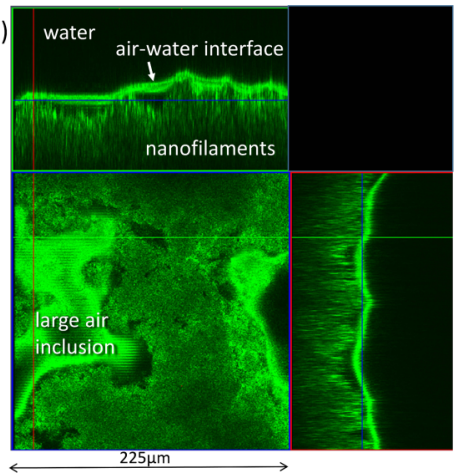

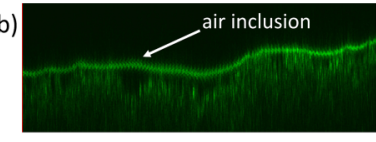

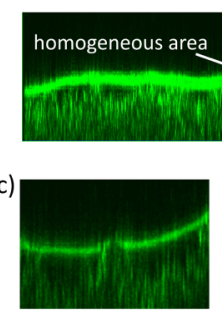

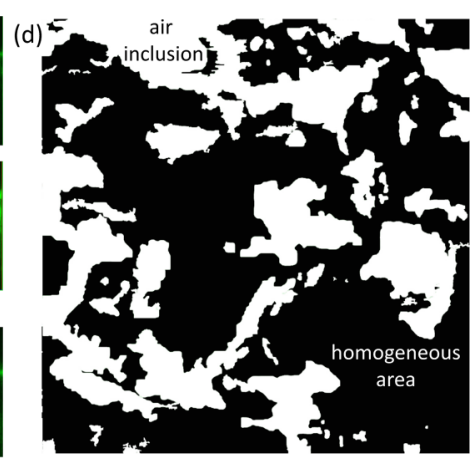

FIG. 3. Confocal imaging of the superhydrophobic coating (reflection channel). The water-air interface appears as a bright line. (a) Cut planes from a 3D image showing a very large air inclusion; (b) exemplary cut sections with typical sizes of air inclusions; (c) interruption in the reflection signal of the air-water interface; (d) analysis of the wetting areas (image width: $225 \mu \mathrm{m}$ ). 
While, on the one hand, the model for a partly immersed detection volume is obtained through a lengthy, formal mathematically derivation, the comparison of its mathematical form with the fully immersed case, on the other hand, leads to an alternative and very intuitive interpretation. For a partly immersed detection volume, we can use the classical fit equation for a fully immersed volume but allocate the measured velocity not to the center of the detection volume (as is typically done) but instead to a position that corresponds to an average of the part of the detection volume that is still immersed in the fluorescent fluid. Then, $U_{\text {eff }}$ can be understood as a velocity at an effective center of the partly immersed detection volume, influenced by its size and distribution [Fig. 2(c)].

To evaluate the quality of the analytical approximation in Eq. (2), we solved the general expression of the FCS autocorrelation function numerically for a partly immersed detection volume. Both solutions show excellent agreement for nearly all immersion fractions of the detection volume [Fig. 2(d)]. Still, in what follows, we restrain ourselves to studying cases in which the detection volume is more than one-third immersed.

Before applying the method to a structured surface, we first verified it on bare, hydrophilic glass. On glass, we expected a no-slip condition at the surface and a parabolic velocity profile throughout the channel, which appears linear upon focusing on a region very close to the surface. As shown in Fig. 2(e), the classical model for a fully immersed volume leads to an artificial deviation from the expected velocity profile in the channel, whereas the model for a partly immersed volume matches the expectation and also well fulfills the no-slip condition within the measurement error of the technique.

To determine the slip length, the precise position of the interface needs to be known. We determined the position of the interface by fitting the detected fluorescence intensity profile recorded across the interface with a cumulative distribution function (CDF) of a Gaussian distribution. The interface position then corresponds to a position where the detection volume is half immersed in a fluorescent medium (see Appendix B).

\section{RESULTS AND DISCUSSION}

\section{A. Wetting situation}

Confocal imaging of the nanofilament coating reveals that the surface exhibits two different regions (Fig. 3): First, homogeneous areas, where the air-water interface uniformly touches the uppermost nanofibers, similar to superhydrophobic microarray surfaces. Second, air inclusions: The superhydrophobicity of the surface may lead to areas where the air-water interface spans across a dip in the surface coating and thereby encapsulates large amounts of air. These air inclusions commonly reach a size of about $50 \times 50 \mu \mathrm{m}$, but may even be larger. Thereby, they exhibit large free-surface sections. While the inner structure of the porous coating as observed with SEM (Fig. 1) suggests typical fiber-fiber distances of about $1 \mu \mathrm{m}$, the air-water interface often spans over much larger distances. There is also a number of smaller air-inclusions of a few $\mu \mathrm{m}$ in size, which may be interconnected with neighboring air inclusions. Altogether, the air inclusions take up an average area fraction of approximately $36 \%$ of the whole surface [evaluation in IMAGEJ, average over six images, considering several $z$ planes for each image, smallest counted inclusion size $\sim 5 \mu \mathrm{m}]$, see Fig. 3(d). Hence, for a significant amount of the surface, the distance between the no-slip conditions the flow is subjected to when moving over the fibers is much larger than suggested by the bulk properties. With respect to the slip properties of the surface, it is thus important to consider the air inclusions. Apart from this, in rare occasions, we find interruptions in the reflection signal of the air-water interface [Fig. 3(c)], which is likely to occur due to individual nanofiber bundles pinching through the air-water interface.

\section{B. Velocity and slip length}

Applying the technique to a nanofilament surface, we measured velocity profiles at the center of air inclusions of various sizes as well as on the homogeneously wetted region. In all cases, we 

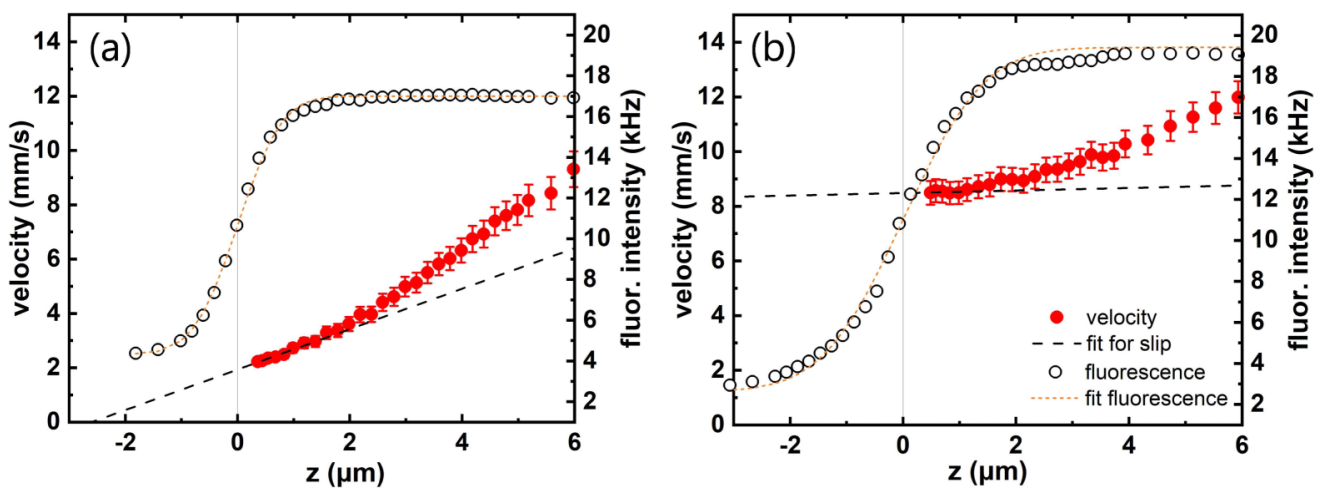

FIG. 4. Velocity profiles perpendicular to the interface (red circles) obtained by fitting the corresponding experimental FCS autocorrelation curves with Eqs. (2) and (3): (a) on the homogeneous porous region and (b) at the center of an air inclusion of about $22 \times 28 \mu \mathrm{m}$. Average fluorescence intensity curves (black, open circles) are fitted with a cumulative distribution function of a Gaussian distribution to detect the position of the interface.

observe that the velocity profiles are still nonlinear at distances below $1 \mu \mathrm{m}$ to the interface (Fig: 4 , more examples in Fig. 5 and Appendix C). This effect is more prominent the faster the velocity at the interface is, i.e., it is stronger on the air inclusions.

For each case, the local slip length was obtained by extrapolating the velocity profile below the interface. For this purpose, a linear function was fitted to the velocity profile within a small range at the end of the velocity profile. Higher order fits are not appropriate because they are not robust and can introduce unphysical slopes in the extrapolated region. Furthermore, there does not exist a universal shape of the velocity profile that is valid on any point on the interface. In general, the function was fitted within a range of $0.4-1 \mu \mathrm{m}$ from the interface.

\section{Slip on air inclusions}

At the center of the air inclusions, the local slip-length values increase strongly with the size of the inclusions (Table I), which is consistent with the expectation that the fluid has more space to accelerate. Since the air inclusions often had an irregular shape and sometimes were connected to each other, the sizes of the air inclusions are to be taken as an orientation, not precise values.
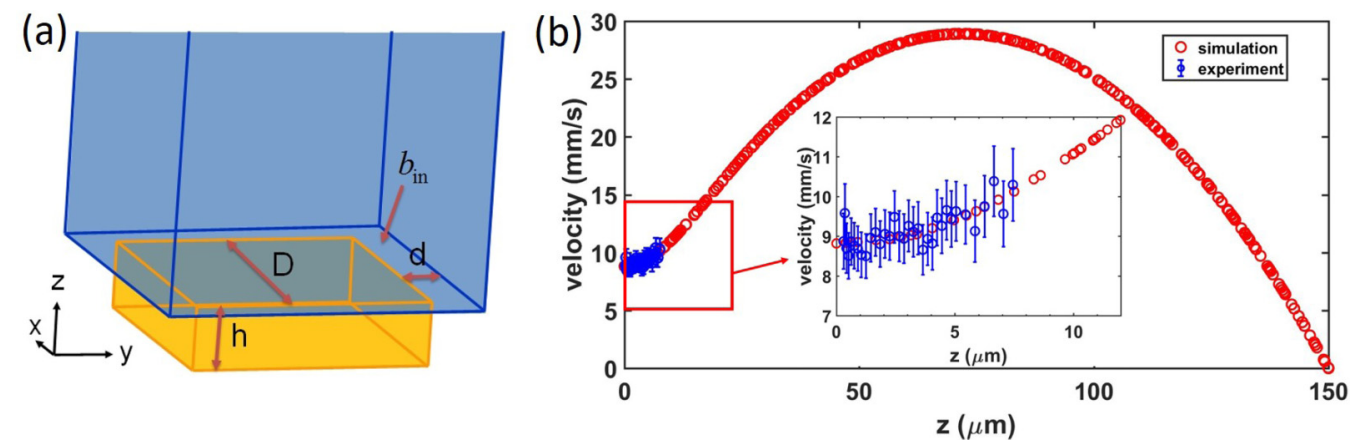

FIG. 5. (a) Sketch of the model of an air inclusion. (b) Exemplary velocity profile at the center of an air inclusion (numerically $D=50 \mu \mathrm{m}$, experimentally $43 \times 47 \mu \mathrm{m}$ ). In the calculations, the pressure gradient was fitted to reproduce the slope of the velocity profile far from the surface 
TABLE I. Experimentally determined local slip-length values at the center of air inclusions of various sizes.

\begin{tabular}{lc}
\hline \hline Size of air inclusion $\left(\mu \mathrm{m}^{2}\right)$ & Local slip length $(\mu \mathrm{m})$ \\
\hline$\sim 43 \times 47$ & 605 \\
$\sim 51 \times 57$ & 576 \\
$\sim 22 \times 28$ & 182 \\
$\sim 12 \times 18$ & 90 \\
$\sim 6 \times 8$ & 88 \\
\hline \hline
\end{tabular}

Furthermore, the nonlinearity of the velocity profile within the last $\mu \mathrm{m}$ close to the surface is important for a reliable measurement of the local slip length. Discarding the velocity values in the last $1 \mu \mathrm{m}$ close to the interface, i.e., using the fully immersed detection volume model of Eq. (1), leads to an underestimation of the local slip length that is the more significant the larger the local slip length is, e.g., discarding the last $\mu \mathrm{m}$ yields on a large air inclusion a slip length of $18 \mu \mathrm{m}$ versus $182 \mu \mathrm{m}$, on a small inclusion 13 versus $30 \mu \mathrm{m}$ and on a tiny inclusion 4 versus $5 \mu \mathrm{m}$ (for examples, see Appendix C).

To compare the experimentally determined slip-length values with theoretical expectations, we developed a simple numerical model of the nanofilament surface including the air inclusions. In the model, the Navier-Stokes equations for both the water and the air are solved using the commercial finite element software COMSOL MULTIPHYSICS. The computational domain consists of a threedimensional periodic element of one air inclusion surrounded by the fiber matrix. For simplicity, the air inclusion was modeled as a square cavity of size $D \times D$ and depth $h$ [Fig. 5(a)]. The fiber matrix had a width $d$ and was modeled as a surface with a slip length of $1 \mu \mathrm{m}$. The height of the channel $H=150 \mu \mathrm{m}$ corresponds to the dimensions of the experiment. It is much larger than the lateral extension of the surface inhomogeneities, such that any variations in the velocity profile originating from the interface are allowed to equilibrate within the computational domain. The flow was driven by a pressure difference across the channel element. The choice of the width of matrix $d$ did not have a significant effect on the magnitude of the slip length at the center of the air inclusions.

The experimental values for the local slip length evaluated with the model for partial immersion agree with the order of magnitude predicted by the basic numerical model of the nanofilament surface (Table II). Both the calculations and the experiments consistently show the change in the nonlinearity of the velocity profile very close to the surface [Fig. 5(b)].

\section{Slip on homogeneously wetted regions}

On the homogeneously wetted parts of the nanofilament surface, where the air-water interface always touches the uppermost fibers, a series of measurements yields a slip length between 1 and $5 \mu \mathrm{m}$, depending on the position with an average of $3 \pm 2 \mu \mathrm{m}$. The measurements vary due to the irregularity of the surface. Since the velocity profiles are quite close to linear for small slip lengths, the difference between the classical method and the one for a partial immersed detection volume is also not large, i.e., about $1 \mu \mathrm{m}$. Hence, it is reasonable that previous measurements on surfaces with

TABLE II. Simulated local slip-length values at the center of air inclusions of size $D \times D$ and depth $h$, surrounded by a homogeneously wetted fiber region of width $d$, modeled by an effective slip length of $1 \mu \mathrm{m}$ on these areas.

\begin{tabular}{lccccccc}
\hline \hline$D(\mu \mathrm{m})$ & 50 & 40 & 30 & 20 & 10 & 5 & 2 \\
$h(\mu \mathrm{m})$ & 10 & 10 & 8 & 5 & 5 & 3 & 1 \\
$d(\mu \mathrm{m})$ & 5 & 5 & 5 & 5 & 5 & 1.5 & 0.5 \\
$b(\mu \mathrm{m})$ & 612 & 344 & 227 & 180 & 82 & 46 & 19 \\
\hline \hline
\end{tabular}


rather small and uniform slip obtained a good agreement between theory and practice, but others, like irregular surfaces, did not.

To compare with the experiments, we also developed a simple numerical model for the homogeneously wetted regions. Effectively, we estimate the average slip length of the homogeneously wetted region to lie in between two limiting cases: a scenario where all fibers are aligned parallel to the flow direction and a scenario where the fibers are all aligned perpendicular to the flow direction. In these cases, the fibers would either present the largest or smallest obstruction to the flow, while in reality, there is a random orientation of the fibers in between these two scenarios. For simplicity, the fibers in the numerical model form regular, periodic arrays (see Appendix D). With the fiber dimensions estimated form SEM images, the numerical calculations predict a slip length in the range $0.25-4.2 \mu \mathrm{m}$.

Obviously, the experimental and numerical values are in the same range. The slip length of the homogeneously wetted regions hence fits with the expectation stemming from regularly structured surfaces. Due to the wetting scenario, these regions, however, only occupy a fraction of the whole surface. The remaining part of the surface (the air inclusions) has a much larger slip length, which can therefore lead to a larger drag reduction.

\section{Effective slip}

The slip on the homogeneously wetted regions also enhances the flow over the air inclusions. In the numerical calculations, a calculation of a superhydrophobic surface with rectangular cavities of $30 \mu \mathrm{m}$ but no-slip walls predicts a local slip length at the center of the inclusion of $200 \mu \mathrm{m}$; substituting the no-slip wall with a nanofilament matrix with $1 \mu \mathrm{m}$ slip increases this local slip length to $226 \mu \mathrm{m}$.

We refrained from measuring an effective slip length of the whole surface or the drag reduction because this is strongly subject to the assumed reference plane [67]. The effective slip length is classically defined with respect to the plane at the tips of the surface structures. For an irregular surface structure, such as the one investigated here, there is, however, not a defined height of the tips. Different choices for the reference plane could be made. Since the slip length is the virtual extension of the velocity profile beyond this reference plane, the definition of the reference plane will directly translate into different values for the effective slip length. The variation of the possible choices of the reference plane is, however, of the same order of magnitude as the expected effective slip length and would not lead to reliable results. The same problem holds for measuring the drag reduction. Drag reduction is also often defined with respect to the tips of the surface coating. This definition gives the largest values for the drag reduction. However, this definition is delicate in microchannels. Here, the coating might take up a significant amount of the cross section of the microchannel. Choosing a reference plane at the tips of the coating would hence correspond to comparing a channel with a coating to a much smaller channel without coating. Since drag overproportionally increases in smaller channels, the larger channel always outplays the smaller one. After all, there is also a flow (of air) within the coating [68]. Another possibility would be to choose the bottom of the coating as a reference plane, i.e., compare a coated and uncoated channel of the same nominal cross section. This choice corresponds to the picture that a certain space is available and that this space can either be used for a drag-reducing coating or can simply be left open for the fluid to flow through. With this approach, the obtained drag reduction will depend on the design of the whole system, like channel height and coating thickness. For example, for a surface with a certain effective slip length, the smaller the coating thickness the larger the drag reduction. In our experimental setup, the coating thickness was about $15 \%$ of the height of the channel, which was needed to be able to clearly distinguish the reflection from the air-water interface from that of the coating-glass interface and thereby to clearly demonstrate the Cassie state. This choice is, however, not suited to obtain a large drag reduction with the given definition. 


\section{CONCLUSIONS}

In summary, we have investigated in detail the local flow phenomena close to an irregularly patterned, nanostructured superhydrophobic surface using FCS and theoretical calculations. Due to the irregularities, large, stable air inclusions occur on the nanostructured surface. These inclusions possess a very large local apparent slip length, in our case up to several hundreds of $\mu \mathrm{m}$. Additionally, it was experimentally shown that for structured surfaces, the velocity profile is still nonlinear below $1 \mu \mathrm{m}$ close to the wall. For a reliable measurement of the local slip length, the resolution of this variation is essential, so an appropriate experimental technique was developed.

Overall, both the presence of air inclusions as well as the so far unresolved velocity profiles at the nanoscale could contribute to the fact that high drag reduction has been reported for irregular, nanostructured superhydrophobic surfaces, while standard theoretical models and existing direct measurements would only predict small values. There are probably more reasons, such as the choice of the reference plane for the evaluation of drag reduction. It is also important for modeling of irregular, superhydrophobic surfaces to realize that they cannot necessarily be considered analogously to a homogeneous porous medium although the bulk of the porous superhydrophobic structure is perfectly equivalent. Deriving a slip boundary condition from the bulk properties only works in the homogeneously wetted areas, which only occupy a fraction of the surface. Another step in modeling would be required to derive an effective slip length from both the homogeneously wetted areas and the air inclusions, where the local slip length was shown to be up to two orders of magnitude larger. These findings may also help in designing new optimized surfaces for high drag reduction.

\section{ACKNOWLEDGMENTS}

The authors thank S. Yordanov and G. Schäfer for helpful discussions on FCS and chemistry. D. Vollmer and M. Kappl are acknowledged for their expertise with the silicone nanofilaments. The authors also thank C. Wei and S.-H. Yuan for providing access to the Chinese Supercomputing Facility. The Chinese Scholarship Council is acknowledged for funding.

\section{APPENDIX A: AUTOCORRELATION FUNCTIONS FOR A PARTLY IMMERSED DETECTION VOLUME}

The autocorrelation function of the fluctuations of a detected fluorescence signal $F(t)$ is

$$
G(\tau)=\frac{\langle\delta F(t) \cdot \delta F(t+\tau)\rangle}{\langle F(t)\rangle^{2}}
$$

where $\delta F(t)=F(t)-\langle F(t)\rangle$, and $\langle\cdot\rangle$ denotes time average. Expressing $F(t)$ in terms of the fluorescent particles concentration $C$ at position $\vec{r}$ and time $t$, we have $[66,69]$

$$
G(\tau)=\frac{\int_{V^{\prime}} \int_{V} W(\vec{r}) W\left(\vec{r}^{\prime}\right)\left\langle\delta\left(C(\vec{r}, t) \cdot \delta\left(C\left(\vec{r}^{\prime}, t+\tau\right)\right)\right\rangle d V d V^{\prime}\right.}{\left\langle\int_{V} W(\vec{r})\langle\delta(C(\vec{r}, t))\rangle d V\right\rangle^{2}},
$$

where $W(\vec{r})$ is a measure of the effective shape of the detection volume and represents the product of the spatial distribution of the focused excitation laser intensity and the optical transfer function of the objective-pinhole combination. For FCS, it is typically assumed to have a three-dimensional Gaussian intensity profile, which is expressed as

$$
W(\vec{r})=e^{-2 \frac{x^{2}+y^{2}}{r_{0}^{2}}} \cdot e^{-2 \frac{z^{2}}{z_{0}^{2}}}
$$

Note that $r_{0}$ and $z_{0}$ are constant, which means that the Gaussian beam is invariant in the $x-y$ plane. This assumption is a simplification due to analytical tractability. It has proven to perform well for FCS measurements as it is the standard assumption behind most models, although a diverging beam would correspond more to the actual profile. 



FIG. 6. Schematic of the detection volume. (a) Fluorescent signal can originate only from the part immersed in water. (b) For mathematical convenience, this part can be divided in two segments $\left(V_{1}\right.$ and $\left.V_{2}\right)$ separated by the centerline $\mathrm{C}$.

In the fully immersed case, the integration in Eq. (A2) is performed over the whole coordinate space. Here, we assume that there are no contributions to the fluorescence signal from medium 2 so the integration is performed only over the region containing the fluorescent fluid, in our application water [Fig. 6(a)]. Other possible influence factors, such as light scattering from the interface, a possible deformation of the detection volume close to the interface or a possible change of the diffusion coefficient close to the interface are neglected for the present model. Since the detection volume is asymmetric in $z$, it is convenient to perform the integration in Eq. (A2) in two parts, $-\infty$ to 0 for $V_{1}$ and 0 to $L$ for $V_{2}$ [Fig. 6(b)].

For the solution of [Eq. (A2)], let us for convenience first define a function $\phi$ as

$$
\phi\left(\vec{r}, \vec{r}^{\prime}, \tau\right)=\left\langle\delta C(\vec{r}, 0) \delta C\left(\vec{r}^{\prime}, \tau\right)\right\rangle=\frac{1}{T} \int_{0}^{T} \delta C(\vec{r}, t) \delta C\left(\vec{r}^{\prime}, t+\tau\right) d t .
$$

Here, $\delta C(\vec{r}, \tau)$ is the fluctuation of the concentration of the diffusing and flowing fluorescent particles as a function of the location $\vec{r}$ and time $\tau$. One can derive it by solving the convection-diffusion equation.

In the following, we consider two cases: a constant flow velocity over the detection volume and a linear flow velocity distribution over the $z$ axis of detection volume. The constant velocity case is the standard assumption used in most previous works for FCS-based flow measurements. It, however, is not necessarily a good representation of the actual situation near interfaces, since the velocity gradient perpendicular to the interface may be significant.

\section{Constant velocity}

The concentration of the diffusing fluorescent particles inside the detection volume $C(\vec{r}, \tau)$ can be described by a convection-diffusion. Since $\delta C(\vec{r}, \tau)=C(\vec{r}, \tau)-\langle C(\vec{r}, \tau)\rangle,(\langle C(\vec{r}, \tau)\rangle$ is the time average concentration, this also holds for the concentration fluctuations $\delta C(\vec{r}, \tau)$. Thus, for a constant velocity $V$ across the detection volume pointing in the $y$ direction, the convection-diffusion equation reads

$$
\frac{\partial \delta C(\vec{r}, \tau)}{\partial \tau}=D \nabla^{2} \delta C(\vec{r}, \tau)-V \cdot \frac{\partial \delta C(\vec{r}, \tau)}{\partial y},
$$

where $D$ is the diffusion coefficient for the particle concentration $C(\vec{r}, \tau)$. The solution of Eq. (A5) in Fourier space is expressed as [69]

$$
\tilde{C}(\nu, \tau)=\tilde{C}(\nu, 0) \cdot e^{-D v^{2} \tau-i v_{y} V \tau},
$$


where $\tilde{C}(v, \tau)$ is the Fourier transformation of $\delta C\left(\vec{r}^{\prime}, \tau\right), \vec{v}=\left(v_{x}, v_{y}, v_{z}\right), v^{2}=v_{x}^{2}+v_{y}^{2}+v_{z}^{2}$. On substitution of Eq. (A6) into Eq. (A4), we arrive at

$$
\begin{aligned}
\phi\left(\vec{r}, \vec{r}^{\prime}, \tau\right) & =F_{r^{\prime}}^{-1}\left(e^{\left(-D v^{2} \tau-i v_{y} V \tau\right)}\langle\delta C(\vec{r}, 0) \tilde{C}(v, 0)\rangle\right) \\
& =F_{r^{\prime}}^{-1}\left(e^{\left(-D v^{2} \tau-i v_{y} V \tau\right)} F_{v}\left\langle\delta C(\vec{r}, 0) \delta C\left(\vec{r}^{\prime \prime}, 0\right)\right\rangle\right) .
\end{aligned}
$$

As the concentration of the fluorescent particles in the detection volume is very small, the particles are assumed to only autocorrelate with themselves. Thus, after averaging over time,

$$
\left\langle\delta C(\vec{r}, 0) \delta C\left(\vec{r}^{\prime \prime}, 0\right)\right\rangle=\bar{C} \delta\left(\vec{r}-\vec{r}^{\prime \prime}\right),
$$

where $\bar{C}$ is the mean-square fluctuation of $\delta C(\vec{r}, t)$ in a unit volume.

A general form of $\delta C(\vec{r}, t)$ in Fourier expansion is

$$
\begin{aligned}
\delta C(\vec{r}, t) & =F_{r}^{-1}(\tilde{C}(\nu, \tau)) \\
& =\frac{1}{4 \pi^{2}} \int_{-\infty}^{+\infty} d v_{x} \int_{-\infty}^{+\infty} d v_{y} \int_{-\infty}^{+\infty} d v_{z} e^{i\left(x v_{x}+y v_{y}+z v_{z}\right)} \tilde{C}(\nu, \tau)
\end{aligned}
$$

where $\tilde{C}(\nu, \tau)=F_{v}(\delta C(\vec{r}, t))=\frac{1}{2 \pi} \int_{-\infty}^{+\infty} d x \int_{-\infty}^{+\infty} d y \int_{-\infty}^{+\infty} d z e^{-i\left(x v_{x}+y v_{y}+z v_{z}\right)} \delta C(\vec{r}, \tau)$.

On substitution of Eq. (A9) into Eq. (A7), $\phi\left(\vec{r}, \vec{r}^{\prime}, \tau\right)$ can be expressed in Cartesian coordinates as

$$
\phi\left(\vec{r}, \vec{r}^{\prime}, \tau\right)=\frac{\bar{C}}{8 \pi^{3}}\left(\sqrt{\frac{\pi}{D \tau}}\right)^{3} e^{-\frac{\left(x-x^{\prime}\right)^{2}+\left(y-y^{\prime}+V \tau\right)^{2}+\left(z-z^{\prime}\right)^{2}}{4 D \tau}} .
$$

Hence, inserting Eq. (A3) and (A10) into Eq. (A2) yields the expression to be evaluated for the autocorrelation function with a finite boundary $L$,

$$
G(\tau, L)=\frac{\int_{-\infty}^{+\infty} d x \int_{-\infty}^{+\infty} d y \int_{-\infty}^{L} d z W(\vec{r}) \int_{-\infty}^{+\infty} d x^{\prime} \int_{-\infty}^{+\infty} d y^{\prime} \int_{-\infty}^{L} d z^{\prime} W\left(\vec{r}^{\prime}\right) \phi\left(\vec{r}, \vec{r}^{\prime}, \tau\right)}{\left(\int_{-\infty}^{+\infty} d x \int_{-\infty}^{+\infty} d y \int_{-\infty}^{L} d z W(\vec{r})\langle\delta(C(\vec{r}, t))\rangle\right)^{2}} .
$$

Note that the perturbation of the fluorescent particle concentration is independent on the position. Thus, $\langle\delta(C(\vec{r}, t))\rangle^{2}=\bar{C}^{2}$. The expression can be expanded in Fourier space,

$$
\begin{aligned}
G(\tau, L, V)= & \frac{1}{8 \pi^{3} \bar{C}}\left(\sqrt{\frac{\pi}{D \tau}}\right)^{3} \times \frac{1}{\left\langle\int_{-\infty}^{+\infty} d x \int_{-\infty}^{+\infty} d y \int_{-\infty}^{L} d z e^{-2 \frac{x^{2}+y^{2}}{r_{0}^{2}}} \cdot e^{\left.-2 \frac{z^{2}}{z_{0}^{2}}\right\rangle^{2}}\right.} \\
& \times\left(\int_{-\infty}^{+\infty} d x \int_{-\infty}^{+\infty} d y \int_{-\infty}^{L} d z \int_{-\infty}^{+\infty} d x^{\prime} \int_{-\infty}^{+\infty} d y^{\prime} \int_{-\infty}^{L} d z^{\prime} e^{-\frac{\left(x-x^{\prime}\right)^{2}+\left(y-y^{\prime}+V \tau\right)^{2}+\left(z-z^{\prime}\right)^{2}}{4 D \tau}}\right. \\
& \left.\times e^{-2 \frac{x^{\prime 2}+y^{\prime 2}}{r_{0}^{2}}} \cdot e^{-2 \frac{z^{\prime 2}}{z_{0}^{2}}} \cdot e^{-2 \frac{x^{2}+y^{2}}{r_{0}^{2}}} \cdot e^{-2 \frac{z^{2}}{z_{0}^{2}}}\right) \\
= & \frac{1}{8 \pi^{3} \bar{C}} \frac{1}{\left\langle\int_{-\infty}^{+\infty} d x \int_{-\infty}^{+\infty} d y \int_{-\infty}^{L} d z e^{-2 \frac{x^{2}+y^{2}}{r_{0}^{2}}} \cdot e^{\left.-2 \frac{z^{2}}{z_{0}^{2}}\right\rangle^{2}}\right.} \\
& \times\left(\int_{-\infty}^{+\infty} d v_{x} \int_{-\infty}^{+\infty} d v_{y} \int_{-\infty}^{+\infty} d v_{z} e^{-D v^{2} \tau} \cdot e^{-i v_{y} V \tau}\right. \\
& \times \int_{-\infty}^{+\infty} d x \int_{-\infty}^{+\infty} d y \int_{-\infty}^{L} d z e^{-2 \frac{x^{2}+y^{2}}{r_{0}^{2}}} \cdot e^{-2 \frac{z^{2}}{z_{0}^{2}}} \cdot e^{-i\left(x v_{x}+y v_{y}+z v_{z}\right)} \\
& \left.\times \int_{-\infty}^{+\infty} d x^{\prime} \int_{-\infty}^{+\infty} d y^{\prime} \int_{-\infty}^{L} d z^{\prime} e^{-2 \frac{x^{\prime 2}+y^{\prime 2}}{r_{0}^{2}}} \cdot e^{-2 \frac{z^{\prime 2}}{z_{0}^{2}}} \cdot e^{i\left(x^{\prime} v_{x}+y^{\prime} v_{y}+z^{\prime} v_{z}\right)}\right)
\end{aligned}
$$




$$
\begin{aligned}
& =\frac{1}{8 \pi^{3} \bar{C}} \frac{\frac{r_{0}^{4}}{4} \pi^{2}}{\left\langle\int_{-\infty}^{+\infty} d x \int_{-\infty}^{+\infty} d y \int_{-\infty}^{L} d z e^{-2 \frac{x^{2}+y^{2}}{r_{0}^{2}}} \cdot e^{\left.-2 \frac{z^{2}}{z_{0}^{2}}\right\rangle^{2}}\right.} \times \int_{-\infty}^{+\infty} e^{-D v_{x}^{2} \tau} e^{-r_{0}^{2} \frac{v_{x}^{2}}{4}} d v_{x} \\
& \times \int_{-\infty}^{+\infty} e^{-D v_{y}^{2} \tau} e^{-i \nu_{y} V \tau} e^{-r_{0}^{2} \frac{\nu_{y}^{2}}{4}} d v_{y} \times \int_{-\infty}^{+\infty} d \nu_{z} e^{-D v_{z}^{2} \tau} \int_{-\infty}^{L} d z e^{-2 \frac{z^{2}}{z_{0}^{2}}} e^{-i z v_{z}} \\
& \times \int_{-\infty}^{L} d z^{\prime} e^{-2 \frac{z^{2}}{z_{0}^{2}}} e^{i z^{\prime} v_{z}} \\
& =\frac{1}{8 \pi^{3} \bar{C}} \frac{\frac{r_{0}^{4}}{4} \pi^{2}}{\left\langle\int_{-\infty}^{+\infty} d x \int_{-\infty}^{+\infty} d y \int_{-\infty}^{L} d z e^{-2 \frac{x^{2}+y^{2}}{r_{0}^{2}}} \cdot e^{-2 \frac{z^{2}}{z_{0}^{2}}}\right\rangle^{2}}\left(\sqrt{\pi} \frac{1}{\sqrt{D \tau+\frac{r_{0}^{2}}{4}}}\right)^{2} \\
& \times e^{-(\tau V)^{2} \frac{1}{4 D \tau+r_{0}^{2}}} \int_{-\infty}^{+\infty} d \nu_{z} e^{-D \nu_{z}^{2} \tau} \int_{-\infty}^{L} d z e^{-2 \frac{z^{2}}{z_{0}^{2}}} \cdot e^{-i z v_{z}} \int_{-\infty}^{L} d z^{\prime} e^{-2 \frac{z^{\prime 2}}{z_{0}^{2}}} \cdot e^{i z^{\prime} \nu_{z}} \\
& =\frac{1}{8 \bar{C}} \frac{r_{0}^{2}}{\left\langle\int_{-\infty}^{+\infty} d x \int_{-\infty}^{+\infty} d y \int_{-\infty}^{L} d z e^{-2 \frac{x^{2}+y^{2}}{r_{0}^{2}}} \cdot e^{\left.-2 \frac{z^{2}}{z_{0}^{2}}\right\rangle^{2}} \frac{1}{\frac{\tau}{\tau_{D}}+1}\right.} \\
& \times e^{-\left(\frac{\tau V}{r_{0}}\right)^{2} \frac{1}{\tau_{D}}+1} \int_{-\infty}^{+\infty} d \nu_{z} e^{-D v_{z}^{2} \tau} \int_{-\infty}^{L} d z e^{-2 \frac{z^{2}}{z_{0}^{2}}} \cdot e^{-i z v_{z}} \int_{-\infty}^{L} d z^{\prime} e^{-2 \frac{z^{2}}{z_{0}^{2}}} \cdot e^{i z^{\prime} v_{z}},
\end{aligned}
$$

where $\tau_{D}=\frac{r_{0}^{2}}{4 D}$, is the diffusion time corresponding to $r_{0}$. Note that $\tau_{D}$ does not change with the position of the detection volume, because it is merely a definition based on $r_{0}$. For convenience, we also define

$$
N(L)=\frac{1}{8 \bar{C}} \frac{r_{0}^{2}}{\left\langle\int_{-\infty}^{+\infty} d x \int_{-\infty}^{+\infty} d y \int_{-\infty}^{L} d z e^{-2 \frac{x^{2}+y^{2}}{r_{0}^{2}}} \cdot e^{\left.-2 \frac{z^{2}}{z_{0}^{2}}\right\rangle^{2}} .\right.}
$$

In practice, this expression is a fitting parameter. Considering that the final expression of $G(\tau, L, V)$ is not a function of $z$ and $v_{z}$ since $z$ is eliminated by the integration up to $L$ and $v_{z}$ is eliminated by integration up to infinity, $G(\tau, L, V)$ is eventually a function of $\tau$ at a certain $L$ and $V$. However, in Eq. (A12), the term with integration with respect to $z$ and boundary $L$ does not have an explicit solution. To nevertheless obtain an explicit expression that can be used for fitting to the experimental data later on, we thus need an approximate function of $\tau$ at a certain $L$ and $V$ that well represents this part. We define the inexplicit part as $H(\tau, L, V)$. For a clear description, we express it in two forms in Fourier space and Cartesian coordinates. The form in Cartesian coordinates will later be used in the numerical calculations. We have

$$
\begin{aligned}
H(\tau, L, V) & =\int_{-\infty}^{+\infty} d v_{z} e^{-D v_{z}^{2} \tau} \int_{-\infty}^{L} d z e^{-2 \frac{z^{2}}{z_{0}^{2}}} \cdot e^{-i z v_{z}} \int_{-\infty}^{L} d z^{\prime} e^{-2 \frac{z^{2}}{z_{0}^{2}}} \cdot e^{i z^{\prime} \nu_{z}} \\
& =\sqrt{\frac{\pi}{D \tau}} \int_{-\infty}^{L} d z e^{-2 \frac{z^{2}}{z_{0}^{2}}} \int_{-\infty}^{L} d z^{\prime} e^{-2 \frac{z^{2}}{z_{0}^{2}}} \cdot e^{\frac{-\left(z-z^{\prime}\right)^{2}}{4 D \tau}} \\
& =\sqrt{\frac{\pi}{D \tau}} \sum_{j=1}^{n} \Delta z e^{\frac{z^{2} z^{2}}{4 D \tau\left(8 D \tau+z_{0}^{2}\right)}-\frac{z^{2}}{4 D \tau}} \int_{-\infty}^{L} d z^{\prime} e^{-2 \frac{z^{2}}{z_{0}^{2}}} \times e^{-\frac{\left(z^{\prime}-\frac{z_{0}^{2} z}{\left.8 D \tau \tau z_{0}^{2}\right)^{2}}\right.}{\frac{4 D \tau z_{0}^{2}}{8 D \tau+z_{0}^{2}}}} .
\end{aligned}
$$

To understand Eq. (A14), let us imagine that the inner integration means that when a particle enters at position $z$, it has many possible appearance positions $z^{\prime}$ inside the detection volume after diffusing for a time $\tau$. Therefore, the autocorrelation for one particle should be the summation of 


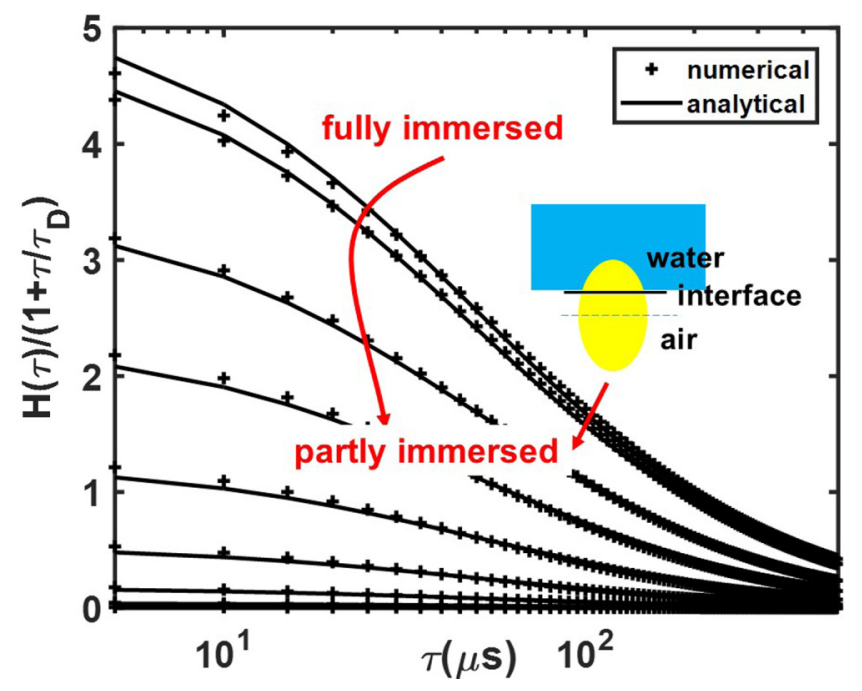

FIG. 7. Comparison between full numerical solution and approximate analytical solution. $L$ is $-1,-0.8$, $-0.6,-0.4,-0.2,0,0.2,0.6,1(\mu \mathrm{m})$, spanning the range from not immersed to fully immersed. The half height of the detection volume is $z_{0}=1 \mu \mathrm{m}$.

all possible $z^{\prime}$, whereas the outer summation for $n$ in Eq. (A14) corresponds to the summation of all autocorrelations for particles that enter at different $z(j=1,2, \cdots, n$ refers to the different positions when entering the detection volume).

Actually, in a partly immersed detection volume, which has a relatively long height compared to the width, the autocorrelation for one particle entering at $z$ does not strongly depend on the height of the detection volume $(\sim L)$ since the overwhelming majority of possible appearance positions $z^{\prime}$ have already been included in the integration range $(-\infty$ to $L)$. In this case, the height of the detection volume is insignificant, since there is almost no difference between a single particle autocorrelation for partial immersion with range from $(-\infty$ to $L)$ and for a full detection volume with range from $(-\infty$ to $\infty)$. Therefore, the inner part is approximately a function of $\tau$ with integration range from $(-\infty$ to $\infty)$, since under infinite integration range, the inner part can be explicitly solved. In this way, the inner and outer integration can be separated. Meanwhile, since the outer summation is related to summing up all particles entering at different positions, the outer summation should be a function of the height of the detection volume, $L$. To sum up, we give an explicit approximate function compared to $H(\tau, L, D)$ as

$$
H(\tau, L, V)=\sqrt{\frac{\pi}{D \tau}} \sum_{j=1}^{n} \Delta z e^{\frac{z_{0}^{2} z^{2}}{4 D \tau \tau\left(8 D \tau+z_{0}^{2}\right)}-\frac{z^{2}}{4 D \tau}} \int_{-\infty}^{L} d z^{\prime} e^{-2 \frac{z^{2}}{z_{0}^{2}}} \times e^{-\frac{\left(z^{\prime}-\frac{z_{0}^{2} z}{8 D \tau+z_{0}^{2}}\right)^{2}}{\frac{4 D \tau \tau}{8 D \tau+z_{0}^{2}}}} \approx f(D, L) T(D, \tau),
$$

where $T(D, \tau)=\frac{z_{0}}{2 \pi^{\frac{1}{2}}} \int_{-\infty}^{+\infty} d \nu_{z} e^{-D v_{z}^{2} \tau} e^{-z_{0}^{2} \frac{v_{z}^{2}}{4}}=\frac{1}{\sqrt{\frac{\tau}{\tau_{D}}\left(\frac{r_{0}}{z_{0}}\right)^{2}+1}}$

To confirm the accuracy and explore the limitation of our approximate explicit function, we compare the autocorrelation function resulting from Eq. (A15) with the autocorrelation function containing the full numerical solution of Eq. (A14) (Fig. 7). We do this for a given list of cases at the same $D$ and $L$. The test conditions are as follows: $z_{0}=1 \mu \mathrm{m}, r_{0}=0.2 \mu \mathrm{m}, D=2^{-10} \mathrm{~m}^{2} / \mathrm{s}$. $L$ is chosen to be $-1,-0.8,-0.6,-0.4,-0.2,0,0.2,0.6,1(\mu \mathrm{m}) . f(D, L)$ is determined by fitting once and constant throughout all $\tau$ cases of each line. In Fig. 7, the approximate analytical expression (lines) fits the full numerical solution (crosses) well, even when there is only less than half of the 
detection volume immersed in the bulk. Remarkably, the choice of $z_{0}$ and $r_{0}$ with $z_{0} / r_{0}=5$ is at the lower end of what can be considered a long, thin detection volume, but which is an experimentally reasonable condition. Larger ratios, e.g., with $z_{0}=2 \mu \mathrm{m}$, even improve the fit.

Ultimately, upon substitution of Eq. (A15) into Eq. (A12), the latter can be further simplified. $f(D, L)$ can be combined with $N(L)$ as a single fitting parameter, that is, $N^{\prime}(D, L)=N(L) \cdot f(D, L)$. $N^{\prime}$ is a complicated fitting parameter which incorporates diffusion and the height of detection volume. Finally, the autocorrelation expression for a constant velocity within a partly immersed detection volume is

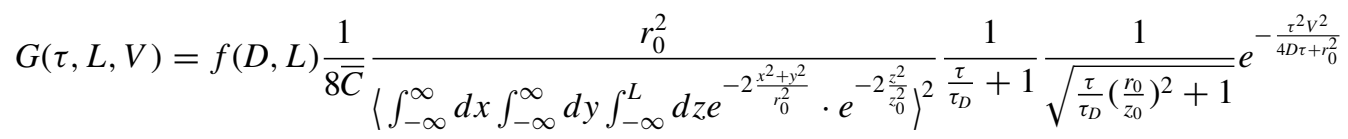

$$
\begin{aligned}
& =N^{\prime}(D, L) \cdot \frac{1}{\frac{\tau}{\tau_{D}}+1} \frac{1}{\sqrt{1+\frac{\tau}{\tau_{D}}\left(\frac{r_{0}}{z_{0}}\right)^{2}}} e^{-\frac{\tau^{2} V^{2}}{r_{0}^{2}} \frac{1}{1+\frac{\tau}{\tau_{D}}}} .
\end{aligned}
$$

Surprisingly, the autocorrelation function for a partly immersed detection volume shares the same form with the expression of a fully immersed detection volume which is the standard method used in previous FCS applications. The difference between these two is that $N$ in the fully immersed case reflects the average number of fluorescent particles in the detection volume, which should be a constant. In contrast, $N^{\prime}$ is a fitting parameter related to $L$. This finding is quite consistent with experimental data. Fitting $N$ in the bulk is always approximately constant, but it changes with $L$ when the detection volume goes across the interface. Regardless of these differences, Eq. (A16) can be used as a uniform expression for velocity fitting at any position of the detection volume across an interface or fully immersed in the bulk.

However, close to an interface, the velocity is not necessarily constant across the detection volume; therefore, in the following part, we will discuss the autocorrelation function for a velocity that is linear in $z$.

\section{Linear velocity distribution}

In this section, the velocity $V(z)$ is assumed to be a linear function within the detection volume.

Let us first explain one assumption. Again, since the particle concentration is very small, we assume that, on average, one particle is present in the detection volume at a certain time $t$. As indicated in Fig. 8, let us imagine that there is a particle entering the detection volume with an initial velocity $V\left(z_{1}\right)$ at some initial position $z$. Since we consider a single particle, $V\left(z_{1}\right)$ is not necessarily equal to the average velocity $V(z)$ at position $z$. Only if we average over all particles entering at height $z$, we obtain the average velocity $V(z)$. The particle shall spend time $\tau$ going through the detection volume. During this time, it moves from $A$ to $B$ due to diffusion. If the flow (convection) is much stronger than diffusion, $\tau$ is comparatively small. This means that the stronger the flow and the slimmer the detection volume, the less time the particle has to accommodate the new flow velocity at position $B$. Thus, even though the particle changes its position from $A$ to $B$, we assume that its velocity does not instantaneously change with a change in $z$ (which would be difficult to assess anyhow, since again, this velocity is not necessarily equal to the average velocity at point $B$ ) but that it maintains its entering velocity $V\left(z_{1}\right)$. This assumption is similar to the assumption of Magde et al. [66] of a long and thin detection volume, such that a particle will diffuse out of the detection volume before it diffuses very far along it. It is necessary to make such an assumption to solve the convection-diffusion equation for the concentration fluctuations. Under experimental conditions, we usually have rather thin detection volumes with $z_{0} / r_{0}>5$ anyhow, as discussed for the constant velocity case.

Under this assumption, the solution of Eq. (A5) can still be written as

$$
\tilde{C}(\nu, \tau)=\tilde{C}(\nu, 0) \cdot e^{-D v^{2} \tau-i v_{y} V\left(z_{1}\right) \tau},
$$




\section{fluorescent particle}

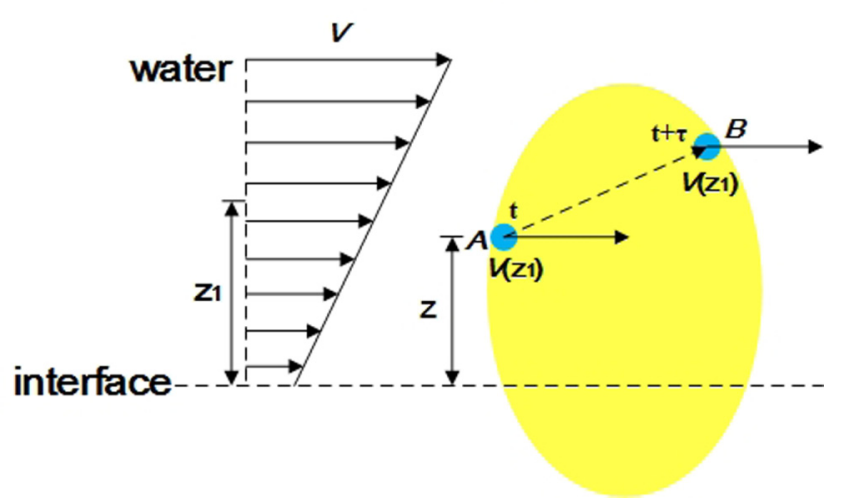

FIG. 8. Motion of particle in detection volume. One particle enters detection volume at $z$ with an initial velocity $V\left(z_{1}\right)$. $z$ is irrelevant to $V\left(z_{1}\right)$. During the motion process in the detection volume, the particle does not change its velocity.

but $V\left(z_{1}\right)$ is the velocity when the particle enters the detection volume at its initial position $z$.

Subsequently, we repeat the process starting from Eq. (A4). We have

$$
\begin{aligned}
\phi\left(\vec{r}, \vec{r}^{\prime}, \tau\right) & =\left\langle\delta C(\vec{r}, 0) \delta C\left(\vec{r}^{\prime}, \tau\right)\right\rangle=F_{r^{\prime}}^{-1}\langle\delta C(\vec{r}, 0) \tilde{C}(\nu, \tau)\rangle \\
& =F_{r^{\prime}}^{-1}\left(e^{\left(-D v^{2} \tau\right)}\left\langle e^{-i v_{y} V\left(z_{1}\right) \tau} \delta C(\vec{r}, 0) \tilde{C}(\nu, 0)\right\rangle\right) .
\end{aligned}
$$

Although the position where the particle enters and its initial velocity $V\left(z_{1}\right)$ may be independent, it is reasonable to believe that all possible values of the velocity in the detection volume will appear if the timescale $T$ is long enough, since the velocity is a $z$-dependent function. Statistically, we have the time average: $\frac{1}{T} \int_{0}^{T} V_{i}\left(z_{1}\right) d t=V(z)(i=1,2, \cdots, N$ refers to the particles entering at the same position at a different time). If we consider two timescales, $T_{1}$ and $T_{2}\left(T_{1} \ll T_{2}\right)$, the evolution in time can be characterized by the two intervals, 0 to $T_{1}$ and 0 to $T_{2}$. During the first time interval, the particle fluctuates due to diffusion and its concentration reaches the mean-square fluctuation concentration but its convective velocity does not yet reach the average value of the new $z$ position. The mean value during this time interval is defined as $V_{i}\left(z_{1}^{\prime}\right)$. Within the longer timescale $\left(0\right.$ to $\left.T_{2}\right)$, the velocity of the particle reaches the equilibrium velocity of the fluid:

$$
\begin{aligned}
\phi\left(\vec{r}, \vec{r}^{\prime}, \tau\right) & =F_{r^{\prime}}^{-1}\left(e^{\left(-D v^{2} \tau\right)} \frac{1}{T_{2}} \int_{0}^{T_{2}} d T_{1} \frac{1}{T_{1}} \int_{0}^{T_{1}} e^{-i v_{y} V\left(z_{1}\right) \tau} \delta C(\vec{r}, 0) \tilde{C}(v, 0) d t\right) \\
& =F_{r^{\prime}}^{-1}\left(e^{\left(-D v^{2} \tau\right)} F_{\nu\left(\vec{r}^{\prime \prime}\right)} \bar{C} \delta\left(\vec{r}-\vec{r}^{\prime \prime}\right) \frac{1}{T_{2}} \int_{0}^{T_{2}} d T_{1} e^{-i v_{y} V_{i}\left(z_{1}^{\prime}\right) \tau}\right) \\
& =F_{r^{\prime}}^{-1}\left(e^{\left(-D v^{2} \tau\right)} e^{-i v_{y} V(z) \tau} F_{\nu\left(\vec{r}^{\prime \prime}\right)} \bar{C} \delta\left(\vec{r}-\vec{r}^{\prime \prime}\right)\right) .
\end{aligned}
$$

In Eq. (A19), any items with constant $V\left(z_{1}\right)$ in one state $\vec{r}$ can be moved out of the time integration and become $V(z)$. Thus, Eq. (A19) is similar to Eq. (A7), except that $V(z)$ is not a constant parameter. 
If we follow the step from Eq. (A10) to Eq. (A11), a similar expression results:

$$
\begin{aligned}
& G(\tau, L, D)=\frac{1}{8 \bar{C}} \frac{r_{0}^{2}}{\left\langle\int_{-\infty}^{+\infty} d x \int_{-\infty}^{+\infty} d y \int_{-\infty}^{L} d z e^{-2 \frac{x^{2}+y^{2}}{r_{0}^{2}}} \cdot e^{\left.-2 \frac{z^{2}}{z_{0}^{2}}\right\rangle^{2}} \frac{1}{\frac{\tau}{\tau_{D}}+1}\right.} \\
& \times \int_{-\infty}^{+\infty} d v_{z} e^{-D v_{z}^{2} \tau} \int_{-\infty}^{L} d z e^{-\frac{\tau^{2} V(z)^{2}}{4 D \tau+r_{0}^{2}}} e^{-2 \frac{z^{2}}{z_{0}^{2}}} \cdot e^{-i z v_{z}} \int_{-\infty}^{L} d z^{\prime} e^{-2 \frac{z^{2}}{z_{0}^{2}}} \cdot e^{i z^{\prime} v_{z}} .
\end{aligned}
$$

Note that $V(z)$ here comes from a statistical (average) result but not a single event. It is clearer to write it as $\frac{1}{N} \sum_{i=1}^{N} V_{i}\left(z_{1}\right)=V(z)(i=1,2, \cdots, N$ refers to the particles entering at the same position at a different time). Now, considering Eqs. (A20) and (A12), we similarly define that

$$
H(\tau, L, V, D)=\sqrt{\frac{\pi}{D \tau}} \int_{-\infty}^{L} d z e^{-2 \frac{z^{2}}{z_{0}^{2}}} \cdot e^{-\frac{\tau^{2}\left(\frac{1}{N} \sum_{i=1}^{N} V_{i}\left(z_{1}\right)\right)^{2}}{4 D \tau+r_{0}^{2}}} \int_{-\infty}^{L} d z^{\prime} e^{-2 \frac{z^{\prime 2}}{z_{0}^{2}}} \cdot e^{\frac{-\left(z-z^{\prime}\right)^{2}}{4 D \tau}} .
$$

To understand the role of particles arbitrarily entering the detection volume with different velocities during the autocorrelation process, we rewrite Eq. (A21) as the summation form:

$$
\begin{aligned}
H(\tau, L, V, D) & =\sqrt{\frac{\pi}{D \tau}} \int_{-\infty}^{L} d z e^{-2 \frac{z^{2}}{z_{0}^{2}}} \cdot e^{-\frac{\tau^{2}\left(\frac{1}{N} \sum_{i=1}^{N} V_{i}\left(z_{1}\right)\right)^{2}}{4 D \tau+r_{0}^{2}}} \int_{-\infty}^{L} d z^{\prime} e^{-2 \frac{z^{\prime 2}}{z_{0}^{2}}} \cdot e^{\frac{-\left(z-z^{\prime}\right)^{2}}{4 D \tau}} \\
& =\sqrt{\frac{\pi}{D \tau}} \sum_{j=1}^{n} e^{-2 \frac{z^{2}}{z_{0}^{2}}} \cdot e^{-\frac{\tau^{2}\left(\frac{1}{N} \sum_{i=1}^{N} V_{i j}\left(z_{1}\right)\right)^{2}}{4 D \tau+r_{0}^{2}}} \Delta z \int_{-\infty}^{L} d z^{\prime} e^{-2 \frac{z^{2}}{z_{0}^{2}}} \cdot e^{\frac{-\left(z-z^{\prime}\right)^{2}}{4 D \tau}} \\
& =\sqrt{\frac{\pi}{D \tau}} \sum_{j=1}^{n} e^{-2 \frac{z^{2}}{z_{0}^{2}}} \cdot e^{-\frac{\tau^{2}\left(\frac{1}{N} \sum_{i=1}^{N} V_{i j}\left(z_{1}\right)\right)^{2}}{4 D \tau+r_{0}^{2}}} e^{\frac{z_{0}^{2} z^{2}}{4 D \tau \tau\left(8 D \tau+z_{0}^{2}\right)}-\frac{z^{2}}{4 D \tau}} \Delta z \int_{-\infty}^{L} d z^{\prime} e^{-\frac{\left(z^{\prime}-\frac{z_{0}^{2} z}{\left.8 D \tau \tau z_{0}^{2}\right)^{2}}\right.}{\frac{4 D \tau z_{0}^{2}}{8 D \tau+z_{0}^{2}}}} .
\end{aligned}
$$

To understand Eq. (A22), similarly, let us imagine that the inner integration means that when a particle enters at $z$ with $V_{i}\left(z_{1}\right)$, it has many possible positions $z^{\prime}$ inside the detection volume after diffusing for time $\tau$. Therefore, the autocorrelation for one particle should be the summation of all possible $z^{\prime}$, wherein the outer summation over $n$ corresponds to the summation of all autocorrelations for particles entering at different $z$ with $V_{i j}\left(z_{1}\right)(j=1,2, \cdots, n$ refers to the different positions when entering the detection volume). Over a long time, $V_{i j}\left(z_{1}\right)$ becomes $V_{j}(z)$.

Similarly, we can still apply the argument used in Eq. (A15) to obtain an approximate explicit expression of Eq. (A22). If we consider an individual particle, it has a certain probability to appear at position $z$. If this position happens to be within the detection volume, the upper integration boundary is not significant and there is no difference between a fully and partly immersed volume. The integration boundary could then, in principle, be replaced by infinity. For a partly immersed volume, however, the probability of a certain entering position is different from a fully immersed volume.

Thus, we replace $\int_{-\infty}^{L} d z^{\prime} e^{-\frac{\left(z^{\prime}-\frac{z_{0}^{2} z}{8 D \tau+z_{0}^{2}}\right)^{2}}{\frac{4 D \tau z_{0}^{2}}{8 D \tau+z_{0}^{2}}}}$ by $A(D, L) \times \int_{-\infty}^{+\infty} d z^{\prime} e^{-\frac{\left(z^{\prime}-\frac{z_{0}^{2}}{8 D \tau+z_{0}^{2}}\right)^{2}}{\frac{4 D \tau z_{0}^{2}}{8 D \tau+z_{0}^{2}}}}$, in which $A(D, L)$ is a constant that compensates for the different entering probabilities. In this way, $z$ disappears from the inner integration and the two integrations are separated. $e^{-2 \frac{z^{2}}{z_{0}^{2}}} \cdot e^{-\frac{\tau^{2}\left(\frac{1}{N} \sum_{i=1}^{N} V_{i j}\left(z_{1}\right)\right)^{2}}{4 D \tau+r_{0}^{2}}} e^{\frac{z_{0}^{2} z^{2}}{4 D \tau\left(8 D \tau+z_{0}^{2}\right)}-\frac{z^{2}}{4 D \tau}}$ can be regarded as a weight function which represents that the autocorrelation of every individual particle is different and thus contributes differently to the overall autocorrelation function. It contains velocity 
and intensity information. Thus, we arrive at the expression

$$
H(\tau, L, V, D) \approx A(D, L) \times \sqrt{\frac{\pi}{D \tau}} \int_{-\infty}^{L} d z e^{-2 \frac{z^{2}}{z_{0}^{2}}} \cdot e^{-\frac{\tau^{2} V(z)^{2}}{4 D \tau+r_{0}^{2}}} e^{\frac{z_{0}^{2} z^{2}}{4 D \tau\left(8 D \tau+z_{0}^{2}\right)}-\frac{z^{2}}{4 D \tau}} \int_{-\infty}^{+\infty} d z^{\prime} e^{-\frac{\left(z^{\prime}-\frac{z_{0}^{2} z^{2}}{8 D \tau+z_{0}^{2}}\right)^{2}}{\frac{4 D \tau \tau=0}{8 D \tau+z_{0}^{2}}}}
$$

Note that here we again use the average velocity $V(z)$.

To reason this a bit more intricate assumption, we check this assumption for different timescales, $\tau<\tau_{D}, \tau \sim \tau_{D}$ and $\tau>\tau_{D}$ ( $\tau$ is the average time that particles need to pass the detection volume). In the case of $\tau<\tau_{D}$, since $\tau_{D}=r_{0}^{2} / 4 D$, and $r_{0} \sim 0.2 z_{0}$, we can simplify $8 D \tau \ll z_{0}^{2}\left(z_{0}^{2} / r_{0}^{2} \sim 25\right)$. And since $\int_{-\infty}^{L} d z^{\prime} e^{-\frac{\left(z^{\prime}-\frac{z_{0}^{2} z}{\left.8 D \tau+z_{0}^{2}\right)^{2}}\right.}{\frac{4 D \tau z_{0}^{2}}{8 D \tau+z_{0}^{2}}}} \approx \int_{-\infty}^{L} d z^{\prime} e^{\frac{-\left(z-z^{\prime}\right)^{2}}{4 D \tau}} \rightarrow \sqrt{4 \pi D \tau}$, it is clear that the standard deviation of $e^{\frac{-\left(z-z^{\prime}\right)^{2}}{4 D \tau}}$ is $\sigma=\sqrt{2 D \tau} \ll z_{0}$. Hence, if the integration range covers a $2 \sim 3 \sigma$ range around the symmetry axis of the function, the majority of particles entering at different $z$ have been considered.

We thus have for most entering positions $z, \int_{-\infty}^{L} d z^{\prime} e^{-\frac{\left(z^{\prime}-\frac{z_{0}^{2} z^{2}}{8 D \tau+z_{0}^{2}}\right)^{2}}{\frac{4 D \tau z_{0}^{2}}{8 D \tau+z_{0}^{2}}}} \rightarrow \int_{-\infty}^{+\infty} d z^{\prime} e^{-\frac{\left(z^{\prime}-\frac{z_{0}^{2} z}{8 D \tau+z_{0}^{2}}\right)^{2}}{\frac{4 D \tau z_{0}^{2}}{8 D \tau+z_{0}^{2}}}}$. In the case of $\tau>\tau_{D}$, the exact mathematical form has little implications, since the whole autocorrelation $G(\tau, L, D)$ becomes zero [since $W\left(\vec{r}^{\prime}\right)$ becomes zero]. Therefore, the exact format of $\int_{-\infty}^{L} d z^{\prime} e^{-\frac{\left(z^{\prime}-\frac{z_{0}^{2} z}{8 D \tau+z_{0}^{2}}\right)^{2}}{\frac{4 D \tau z_{0}^{2}}{8 D \tau+z_{0}^{2}}}}$ does not matter very much and can thus be replaced by $\int_{-\infty}^{+\infty} d z^{\prime} e^{-\frac{\left(z^{\prime}-\frac{z_{0}^{2} z}{8 D \tau+z_{0}^{2}}\right)^{2}}{\frac{4 D \tau z_{0}^{2}}{8 D \tau+z_{0}^{2}}}}$, which is independent of $z$. For the timescale, $\tau \sim \tau_{D}$, we can estimate $\frac{z^{2}}{4 D \tau}<\frac{z_{0}^{2}}{4 D \tau} \sim 25<\infty(z<$ $z_{0}$ ) for typical conditions. Only when $z<z_{0}$, it significantly contributes to the autocorrelation curve. Here, $\sigma=\sqrt{\frac{2 D \tau z_{0}^{2}}{8 D \tau+z_{0}^{2}}} \sim \sqrt{\frac{100 D \tau}{54}}<\sqrt{\frac{25}{54}} r_{0} \sim 0.14 z_{0}$. Thus there are still many particles entering at different $z$ that under the condition of a long detection volume satisfy $\int_{-\infty}^{L} d z^{\prime} e^{-\frac{\left(z^{\prime}-\frac{z_{0}^{2} z^{2}}{\left.8 D \tau+z_{0}^{2}\right)^{2}}\right.}{\frac{4 D \tau z_{0}^{2}}{8 D \tau+z_{0}^{2}}}} \rightarrow$ $\int_{-\infty}^{+\infty} d z^{\prime} e^{-\frac{\left(z^{\prime}-\frac{z_{0}^{2} z}{8 D \tau+z_{0}^{2}}\right)^{2}}{\frac{4 D \tau z_{0}^{2}}{8 D \tau+z_{0}^{2}}}}$ ticles, the correction factor $A(D, L)$ is added.

Before we give a simpler expression, which can be easily applied in experiments, we need to continue to analyze Eq. (A23). From the discussion in the last paragraph, we can expect that the term $e^{\frac{z_{0}^{2} z^{2}}{4 D \tau\left(8 D \tau+z_{0}^{2}\right)}-\frac{z^{2}}{4 D \tau}}$ may approach a constant since $8 D \tau<z_{0}^{2}$ holds. We explain this in the following. Similarly, we will discuss it for three timescales, $\tau<\tau_{D}, \tau \sim \tau_{D}$, and $\tau>\tau_{D}$. Since $\tau_{D}=r_{0}^{2} / 4 D$, and $r_{0} \sim 0.2 z_{0}$, we can simplify $8 D \tau \ll z_{0}^{2}\left(z_{0}^{2} / r_{0}^{2} \sim 25\right)$. Therefore, an approximation of $e^{\frac{z^{2} z^{2}}{4 D \tau\left(8 D \tau+z_{0}^{2}\right)}-\frac{z^{2}}{4 D \tau}} \rightarrow e^{0}=1$ can be appropriate for a timescale $\tau<\tau_{D}$. For a timescale $\tau>\tau_{D}$, the autocorrelation essentially disappears, since the particle has moved out of the detection volume. Then, the form of $H(\tau, L, V, D)$ is no longer important, since we only need to satisfy the value of $G(\tau, L, D)$ to be zero. Thus, we can still use the approximation of $e^{\frac{z_{0}^{2} z^{2}}{4 D \tau\left(8 D \tau+z_{0}^{2}\right)}-\frac{z^{2}}{4 D \tau}} \sim 1$. For the timescale $\tau \sim \tau_{D}$, we can estimate $\frac{z^{2}}{4 D \tau}<\frac{z_{0}^{2}}{4 D \tau} \sim 25<\infty$ for typical conditions. Only when $z<z_{0}$, the entering particles contribute significantly to the autocorrelation curve. Note that the larger 
the entering position $z$, the less strong the contribution of a particle to the autocorrelation curve. Therefore, we still use $e^{\frac{z_{0}^{2} z^{2}}{4 D \tau\left(8 D \tau+z_{0}^{2}\right)}-\frac{z^{2}}{4 D \tau}} \sim 1$.

Thus, the difficulty to find an applicable expression lies in the term $e^{-\frac{\tau^{2} V(z)^{2}}{4 D \tau+r_{0}^{2}}}$, which contains both $\tau$ and $z$. Assuming a linear velocity close to the interface, which satisfies $V(z)=V_{0}+a z$, we expand this term in Taylor series, $e^{-\frac{\tau^{2} V(z)^{2}}{4 D \tau+r_{0}^{2}}}=e^{-\frac{\tau^{2} V_{0}^{2}}{4 D \tau+r_{0}^{2}}} \cdot e^{-\frac{\tau^{2}\left(2 V_{0} a z+(a z)^{2}\right)}{4 D \tau+r_{0}^{2}}}=e^{-\frac{\tau^{2} V_{0}^{2}}{4 D \tau+r_{0}^{2}}} \times\left(1-\frac{\tau^{2}\left(2 V_{0} a z+(a z)^{2}\right)}{4 D \tau+r_{0}^{2}}+\right.$ $\left.\frac{1}{2}\left(\frac{\tau^{2}\left(2 V_{0} a z+(a z)^{2}\right)}{4 D \tau+r_{0}^{2}}\right)^{2}-\ldots\right)$. If $a$ is small (large slip), $V(z) \sim V_{0}$. If $a$ is not small, we assume that the flow is so fast that the time a particle needs to cross the detection volume by flow $\tau_{\text {flow }}$ is shorter than the time it would need to cross the detection volume by diffusion alone: $\tau_{\text {flow }}<\tau_{D}$. This is fulfilled by the experiments as the lowest velocities are about $1 \mathrm{~mm} / \mathrm{s}$. With $r_{0} \approx 0.2 \mu \mathrm{m}$, we can estimate $0.2 \mu \mathrm{m} / \frac{1 \mathrm{~mm}}{\mathrm{~s}}=200 \mu \mathrm{s}<\tau_{D} \approx 440 \mu \mathrm{s}$. Hence, $\frac{\tau^{2}\left(2 V_{0} a z+(a z)^{2}\right)}{4 D \tau+r_{0}^{2}}<1$ and we approximate $e^{-\frac{\tau^{2} V(z)^{2}}{4 D \tau+r_{0}^{2}}}=e^{-\frac{\tau^{2} V_{0}^{2}}{4 D \tau+r_{0}^{2}}} \cdot e^{-\frac{\tau^{2}\left(2 V_{0} a z+(a z)^{2}\right)}{4 D \tau+r_{0}^{2}}} \approx e^{-\frac{\tau^{2} V_{0}^{2}}{4 D \tau+r_{0}^{2}}} \times\left(1-\frac{\tau^{2}\left(2 V_{0} a z+(a z)^{2}\right)}{4 D \tau+r_{0}^{2}}\right)$. Under these conditions, $\tau$ and $z$ in Eq. (A23) can be separated completely. Thus, there must exist a constant velocity $\bar{V}$ that yields the same autocorrelation as $V(z)$ for a given position:

$$
H(\tau, L, V(z), D) \approx H(\tau, L, \bar{V}, D) \Rightarrow \int_{-\infty}^{L} d z e^{-2 \frac{z^{2}}{z_{0}^{2}}} \cdot e^{-\frac{\tau^{2} V(z)^{2}}{4 D \tau+r_{0}^{2}}}=e^{-\frac{\tau^{2} \bar{V}^{2}}{4 D \tau+r_{0}^{2}}} \int_{-\infty}^{L} d z e^{-2 \frac{z^{2}}{z_{0}^{2}}}
$$

In the end, we aim at fitting the theoretical autocorrelation curve to the experimentally recorded one. For this purpose, it is much easier to only work with an effective velocity of the partly immersed detection volume that is recorded during one measurement at a specific position of the detection volume than to consider the full variation of the velocity profile across the detection volume. The latter would involve the explicit knowledge of the velocity gradient. Assessing this gradient would require the knowledge of the velocity at a neighboring measurement position and would therefore couple all evaluations of all autocorrelation curves at each measurement position. It is therefore much more practical to be able to instantaneously obtain an effective velocity for each measurement position. We just need to know which position $z_{\text {eff }}$ within the detection volume to allocate this effective velocity to. For a fully immersed detection volume, the measured velocity is always allocated to the center of the detection volume. For a partly immersed detection volume, this does not hold any more. We rather have to find the center of the immersed part of the detection volume. Here, the constant velocity $\bar{V}$ can be interpreted just as an effective velocity, which we define as $\bar{V}=V_{0}+a z_{\text {eff }}$.

Now the remaining task is to find the effective position $z_{\text {eff }}$. To do this, we approximate the term $e^{-\frac{\tau^{2} V(z)^{2}}{4 D \tau+r_{0}^{2}}}$ in Eq. (A24). Again, we consider $\tau_{\text {flow }}<\tau_{D}$ and $\left(V_{0}+a z_{0}\right) \tau_{\text {flow }} \sim r_{0}$. Hence, we expand $e^{-\frac{\tau^{2} V(z)^{2}}{4 D \tau \tau r_{0}^{2}}}=e^{-\frac{\tau^{2} V_{0}^{2}}{4 D \tau+r_{0}^{2}}} \times\left(1-\frac{\tau^{2}\left(2 V_{0} a z+(a z)^{2}\right)}{4 D \tau+r_{0}^{2}}+\ldots\right)$. If $2 V_{0} \gg a z_{0}$, this expression further simplifies to $e^{-\frac{\tau^{2} V_{0}^{2}}{4 D \tau+r_{0}^{2}}} \times\left(1-\frac{\tau^{2} 2 V_{0} a z}{4 D \tau+r_{0}^{2}}+\ldots\right)$. If $V_{0} \sim a z_{0}$ or $V_{0} \ll a z_{0}$, note that $\left(-\frac{\left.\tau^{2}(a z)^{2}\right)}{4 D \tau+r_{0}^{2}}+\frac{1}{2}\left(\frac{\tau^{2}\left(2 V_{0} a z+(a z)^{2}\right)}{4 D \tau+r_{0}^{2}}\right)^{2}-\right.$ $\ldots) \sim O(0)$, since the negative $a z$ term decreases the value of the following expression. Thus, a reasonable approximation is

$$
\begin{aligned}
& e^{-\frac{\tau^{2} V(z)^{2}}{4 D \tau+r_{0}^{2}}} \approx e^{-\frac{\tau^{2} V_{0}^{2}}{4 D \tau+r_{0}^{2}}} \times\left(1-\frac{\tau^{2} 2 V_{0} a z}{4 D \tau+r_{0}^{2}}\right), \\
& \text { and } e^{-\frac{\tau^{2} \bar{V}^{2}}{4 D \tau+r_{0}^{2}}} \approx e^{-\frac{\tau^{2} V_{0}^{2}}{4 D \tau+r_{0}^{2}}} \times\left(1-\frac{\tau^{2} 2 V_{0} a z_{\mathrm{eff}}}{4 D \tau+r_{0}^{2}}\right) \text { with } \bar{V}=V_{0}+a z_{\mathrm{eff}} .
\end{aligned}
$$


Substituting Eq. (A25) into Eq. (A24), we obtain

$$
\int_{-\infty}^{L} a z e^{-2 z^{z^{2}}} d z=a z_{\mathrm{eff}} \int_{-\infty}^{L} e^{-2 \frac{z^{2}}{z_{0}^{2}}} d z
$$

Finally, the position for the effective velocity $\bar{V}$ is

$$
z_{\mathrm{eff}} \approx \frac{a \int_{-\infty}^{L} z e^{-2 \frac{z^{2}}{z_{0}^{2}}} d z}{a \int_{-\infty}^{L} e^{-2 \frac{z^{2}}{z_{0}^{2}}} d z}=\frac{\frac{z_{0}^{2}}{4} e^{-2 \frac{L^{2}}{z_{0}^{2}}}}{\int_{-\infty}^{L} e^{-2 \frac{z^{2}}{z_{0}^{2}}} d z}
$$

Now, if we replace $e^{-\frac{\tau^{2} V(z)^{2}}{4 D \tau+r_{0}^{2}}}$ with $e^{-\frac{\tau^{2} \bar{V}^{2}}{4 D \tau+r_{0}^{2}}}$ Eq. (A21) becomes

$$
H(\tau, L, V, D) \approx H(\tau, L, \bar{V}, D)=\sqrt{\frac{\pi}{D \tau}} e^{-\frac{\tau^{2} \bar{V}^{2}}{4 D \tau+r_{0}^{2}}} \int_{-\infty}^{L} d z e^{-2 \frac{z^{2}}{z_{0}^{2}}} \int_{-\infty}^{L} d z^{\prime} e^{-2 \frac{z^{2}}{z_{0}^{2}}} \cdot e^{\frac{-\left(z-z^{\prime}\right)^{2}}{4 D \tau}} .
$$

Substituting Eq. (A28) into Eq. (A20), the expression becomes completely analog to the constant velocity case - this assumption enables the calculation of an approximate, explicit autocorrelation function. Upon repeating the process from Eq. (A12) to Eq. (A15), we reach

$$
G(\tau, L, V)=N^{\prime \prime}(D, L) \cdot \frac{1}{\frac{\tau}{\tau_{D}}+1} \frac{1}{\sqrt{1+\frac{\tau}{\tau_{D}}\left(\frac{r_{0}}{z_{0}}\right)^{2}}} e^{-\frac{\tau^{2} \bar{V}^{2}}{r_{0}^{2}} \frac{1}{1+\frac{\tau}{\tau_{D}}}}
$$

where $4 D \tau_{D}=r_{0}^{2}$.

Independently of the multitude of assumptions made, we check the validity and hence applicability of the final expression against numerical solutions of the full, unsimplified expression of the autocorrelation function. This is performed analogously to the constant velocity case. The results are shown in Fig. 2(d) in the main text and show that Eq. (A29) can well represent the autocorrelation function for a partly immersed detection volume. For normalization, we used $G_{0}=\frac{1}{8 \bar{C}} \frac{r_{0}^{2}}{\left\langle\int_{-\infty}^{+\infty} d x \int_{-\infty}^{+\infty} d y \int_{-\infty}^{L} d z e^{-2 \frac{x^{2}+y^{2}}{r_{0}^{2}}} \cdot e^{\left.-2 \frac{z^{2}}{z_{0}^{2}}\right\rangle^{2}}\right.}$.

Although Eq. (A29) shows very good agreement with the numerical solution over the whole range of $L$, it is advisable to apply it only when the detection volume is more than one-third immersed. This is not because of the specific assumptions we made but an observation from experiments. It is likely that below this limit, further effects start to become important, which are considered neither by the full numerical solution nor by the approximate, explicit one. For example, it is likely that the standard assumption of a Gaussian beam starts to become inappropriate beyond this point. The Gaussian beam assumes a constant $r_{0}$, while in reality it will likely increase with the distance from the center of the detection volume. Fitting with a too-small $r_{0}$ would result in an underestimation of the velocity.

\section{APPENDIX B: INTERFACE DETECTION}

The position of the interface was detected through the intensity of the detected fluorescence (average counts). Similar to the arguments described above, as the detection volume moves across a fluidic interface, the fluorescence intensity will go down because only the fluid contains fluorescent tracers (cf. Fig. 9).

With the classical model that the effective shape of the detection volume has a Gaussian shape [Eq. (A3)], integration over the fluorescent region just corresponds to a CDF of a Gaussian function. The position of the interface corresponds to a half-immersed detection volume (Fig. 9). In the experiment, we record the fluorescence intensity in small steps as the detection volume moves in the 
(a)

detection volume fluorescence distribution in Oxyz-coordinate

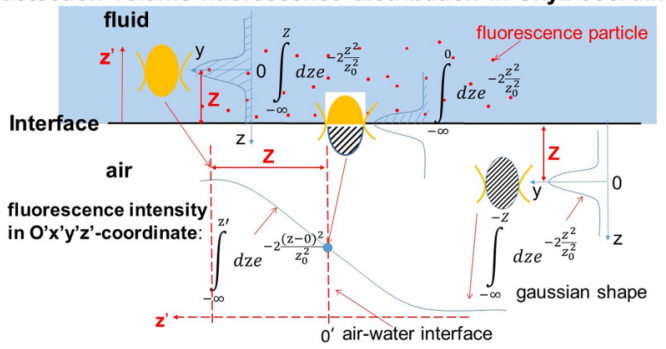

(b)

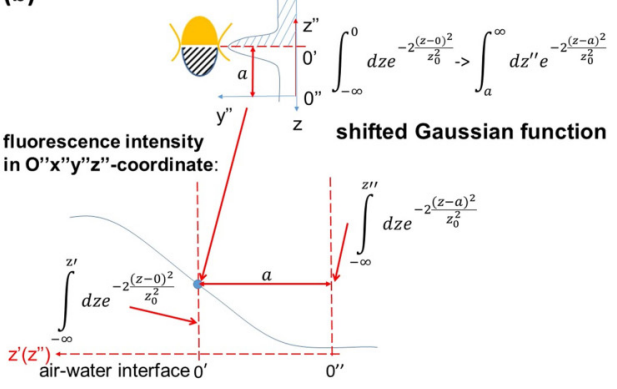

FIG. 9. (a) Position of the detection volume relative to the interface (detection volume is defined to be a normalized Gaussian function); (b) detected fluorescence intensity described by a CDF of Gaussian function. The $x y z$ coordinate system corresponds to the same coordinate that were used for the derivation of the autocorrelation function. The $x^{\prime} y^{\prime} z^{\prime}$ coordinate system is located at the interface as shown in the figure. The $x^{\prime} y^{\prime} z^{\prime}$ coordinates are shifted until the $x^{\prime \prime} y^{\prime \prime} z^{\prime \prime}$ coordinates are obtained, which are the reference coordinates for the experimental values.

$z$ direction across the interface. This curve can be fitted well with the CDF of a Gaussian function:

$$
F(z)=\frac{2}{\sqrt{2 \pi} z_{0}} \int_{-\infty}^{z} \mathrm{~d} x e^{-2 \frac{(x-a)^{2}}{z_{0}^{2}}}+\Pi
$$

The curve is shifted in the $z$ direction via the parameter $a$ until a best fit is obtained. The parameter $a$ then directly gives the position of the interface. The constant $\Pi$ allows for a small offset due to scattered light from the system.

\section{APPENDIX C: EVALUATION OF THE LOCAL SLIP LENGTH}

Figures 10 and 11 show exemplary velocity profiles, where the local slip length is evaluated once with the classical method, i.e., discarding all values below $1 \mu \mathrm{m}$ close to the interface, and once with our method, which provides more values close to the surface.
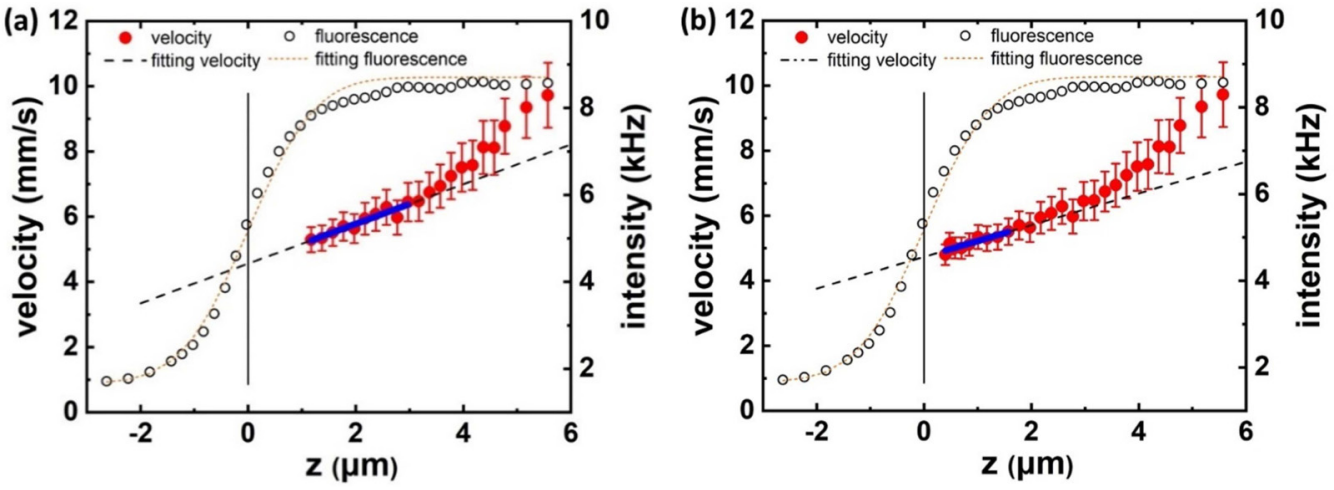

FIG. 10. Example for a small inclusion: (a) classical method, values $<1 \mu \mathrm{m}$ to the surface are discarded, evaluated slip length $b=7.5 \mu \mathrm{m}$; (b) with method for partial immersion, evaluated slip length $b=12 \mu \mathrm{m}$. 

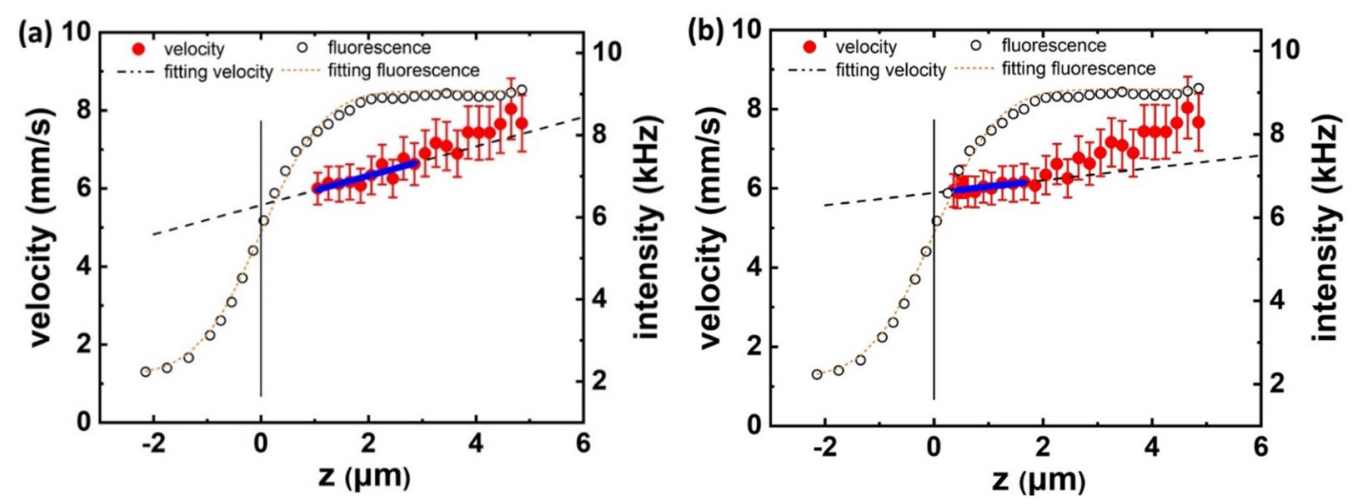

FIG. 11. Example for a medium size inclusion: (a) classical method, values $<1 \mu \mathrm{m}$ to the surface are discarded, evaluated slip length $b=14.8 \mu \mathrm{m}$; (b) with method for partial immersion, evaluated slip length $b=37 \mu \mathrm{m}$.

\section{APPENDIX D: NUMERICAL MODEL FOR ESTIMATING THE SLIP LENGTH OF THE HOMOGENEOUSLY WETTED REGIONS}

Analogously to the simulations for the air inclusions, all simulations were performed by solving the Navier-Stokes equations for the flow of water and air in COMSOL MULTIPHYSICS.

On the homogeneously wetted part of the nanofilament surface, the filaments can have a random orientation. We therefore estimate the actual slip length to lie in between two limiting cases (Fig. 12). In the lower limit, the fibers would form a mesh with air pockets enclosed by the fibers. On the fibers, the slip length is zero. One can also think of this situation as the fibers being oriented in the plane of the interface with a strong connection between the fibers. In this limiting case, the no-slip condition at the fibers is very significant for the flow at the air-water interface as, after passing an air pocket, the water always has to slow again to meet the no-slip condition. In reality, the water is not held back that much by the fibers, and air can move more freely. In the second limiting case, the fibers can be imagined to be oriented perpendicular to the air-water interface. Then the water would only touch the ends of the fibers, and the movement of the air is obstructed very little by the presence of the fibers.

Both patterns are repeated periodically in the $x$ and $y$ directions. From the SEM images, we estimated a typical fiber diameter of $0.5 \mu \mathrm{m}$ and a typical distance of $2 \mu \mathrm{m}$. There is a no-slip

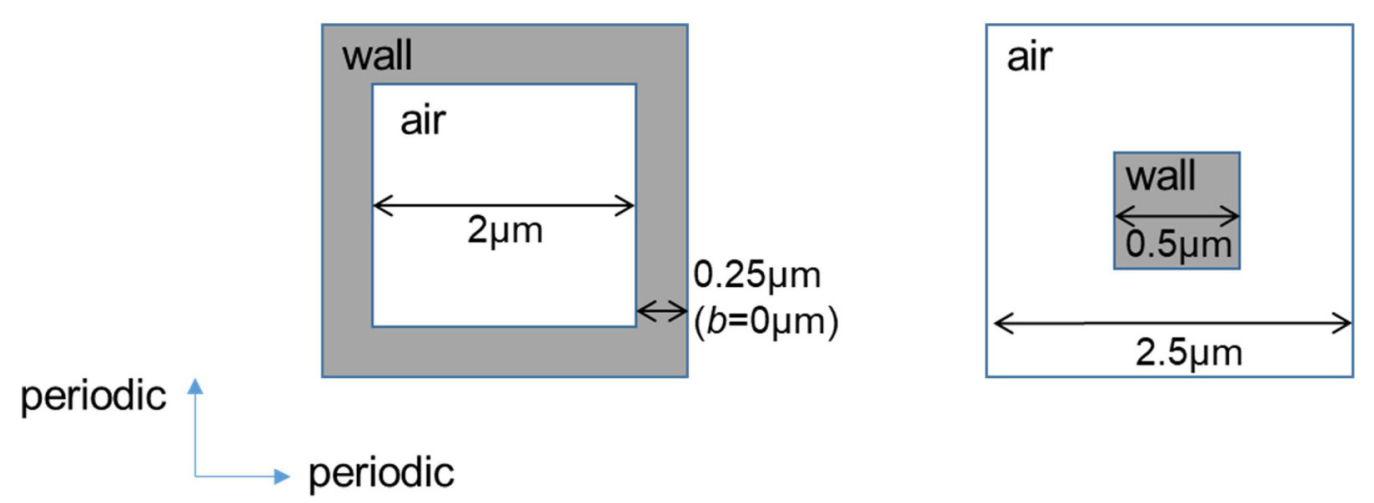

FIG. 12. Top view of the surfaces that define the limiting cases for the calculation of the slip length of the homogeneously wetted areas of the nanofilaments (not to scale). 
boundary condition on the wall section. In all cases, the flow is driven by a velocity of $40 \mathrm{~mm} / \mathrm{s}$ at the upper boundary, ensuring $\operatorname{Re} \ll 1$.

As in the experiments, the finite size of the detection volume leads to a measurement of an average slip length for the homogeneously wetted areas; in the calculations, we correspondingly determine the effective slip length, defined by $b=\langle u\rangle /\langle\partial u / \partial z\rangle$, where $u$ is the velocity in the main flow direction and \langle\rangle denotes the average. In the first limiting case, the slip length calculation yields $b=0.25 \mu \mathrm{m}$ and in the second limiting case $b=4.2 \mu \mathrm{m}$.

[1] A. Nakajima, K. Hashimoto, T. Watanabe, K. Takai, G. Yamauchi, and A. Fujishima, Transparent superhydrophobic thin films with self-cleaning properties, Langmuir 16, 7044 (2000).

[2] R. Fürstner, W. Barthlott, C. Neinhuis, and P. Walzel, Wetting and self-cleaning properties of artificial superhydrophobic surfaces, Langmuir 21, 956 (2005).

[3] M. Ma and R. M. Hill, Superhydrophobic surfaces, Curr. Opin. Colloid Interface Sci. 11, 193 (2006).

[4] B. Bhushan and Y. C. Jung, Natural and biomimetic artificial surfaces for superhydrophobicity, selfcleaning, low adhesion, and drag reduction, Prog. Mater. Sci. 56, 1 (2011).

[5] F. Geyer, M. D’Acunzi, A. Sharifi-Aghili, A. Saal, N. Gao, A. Kaltbeitzel, T.-F. Sloot, R. Berger, H.-J. Butt, and D. Vollmer, When and how self-cleaning of superhydrophobic surfaces works, Sci. Adv. 6, eaaw9727 (2020).

[6] S. Meng, Y. Ye, J. Mansouri, and V. Chen, Fouling and crystallisation behaviour of superhydrophobic nano-composite pvdf membranes in direct contact membrane distillation, J. Membr. Sci. 463, 102 (2014).

[7] L. Schmüser, N. Encinas, M. Paven, D. J. Graham, D. G. Castner, D. Vollmer, H. J. Butt, and T. Weidner, Candle soot-based super-amphiphobic coatings resist protein adsorption, Biointerphases 11, 031007 (2016).

[8] S. Gao, J. Huang, S. Li, H. Liu, F. Li, Y. Li, G. Chen, and Y. Lai, Facile construction of robust fluorinefree superhydrophobic $\mathrm{TiO}_{2}$ fabrics with excellent anti-fouling, water-oil separation and UV-protective properties, Mater. Des. 128, 1 (2017).

[9] K. K. Varanasi, T. Deng, J. D. Smith, M. Hsu, and N. Bhate, Frost formation and ice adhesion on superhydrophobic surfaces, Appl. Phys. Lett. 97, 234102 (2010).

[10] C. Antonini, M. Innocenti, T. Horn, M. Marengo, and A. Amirfazli, Understanding the effect of superhydrophobic coatings on energy reduction in anti-icing systems, Cold Reg. Sci. Technol. 67, 58 (2011).

[11] S. Farhadi, M. Farzaneh, and S. Kulinich, Anti-icing performance of superhydrophobic surfaces, Appl. Surf. Sci. 257, 6264 (2011).

[12] J. C. Bird, R. Dhiman, H.-M. Kwon, and K. K. Varanasi, Reducing the contact time of a bouncing drop, Nature (London) 503, 385 (2013).

[13] Y.-B. Park, H. Im, M. Im, and Y.-K. Choi, Self-cleaning effect of highly water-repellent microshell structures for solar cell applications, J. Mater. Chem. 21, 633 (2011).

[14] F. Geyer, C. Schönecker, H.-J. Butt, and D. Vollmer, Enhancing $\mathrm{CO}_{2}$ capture using robust superomniphobic membranes, Adv. Mater. 29, 1603524 (2017).

[15] R. J. Daniello, N. E. Waterhouse, and J. P. Rothstein, Drag reduction in turbulent flows over superhydrophobic surfaces, Phys. Fluids 21, 085103 (2009).

[16] S. Srinivasan, J. A. Kleingartner, J. B. Gilbert, R. E. Cohen, A. J. B. Milne, and G. H. McKinley, Sustainable Drag Reduction in Turbulent Taylor-Couette Flows by Depositing Sprayable Superhydrophobic Surfaces, Phys. Rev. Lett. 114, 014501 (2015).

[17] G. McHale, M. I. Newton, and N. J. Shirtcliffe, Immersed superhydrophobic surfaces: Gas exchange, slip and drag reduction properties, Soft Matter 6, 714 (2010).

[18] B. Bhushan and M. Nosonovsky, The rose petal effect and the modes of superhydrophobicity, Philos. Trans. R. Soc., A 368, 4713 (2010).

[19] S. Zhang, X. Ouyang, J. Li, S. Gao, S. Han, L. Liu, and H. Wei, Underwater drag-reducing effect of superhydrophobic submarine model, Langmuir 31, 587 (2015). 
[20] B. C. Peifer, C. Callahan-Dudley, and S. A. Mäkiharju, Air layer on superhydrophobic surface for frictional drag reduction, J. Ship Res. 64, 118 (2019).

[21] M. S. Hodes, P. R. Kolodner, T. N. Krupenkin, W. Lee, A. M. Lyons, T. R. Salamon, J. A. Taylor, and D. P. Weiss, Techniques for microchannel cooling, U.S. Patent No. US7204298B2, May, 9th, 2007.

[22] R. Weng, H. Zhang, Y. Tuo, Y. Wang, and X. Liu, Superhydrophobic drag-reduction spherical bearing fabricated by laser ablation and PEI regulated ZnO nanowire growth, Sci. Rep. 7, 6061 (2017).

[23] C. Ybert, C. Barentin, C. Cottin-Bizonne, P. Joseph, and L. Bocquet, Achieving large slip with superhydrophobic surfaces: Scaling laws for generic geometries, Phys. Fluids 19, 123601 (2007).

[24] M. Sbragaglia and A. Prosperetti, A note on the effective slip properties for microchannel flows with ultrahydrophobic surfaces, Phys. Fluids 19, 043603 (2007).

[25] C.-O. Ng and C. Y. Wang, Apparent slip arising from stokes shear flow over a bidimensional patterned surface, Microfluid. Nanofluid. 8, 361 (2010).

[26] A. M. J. Davis and E. Lauga, Hydrodynamic friction of fakir-like superhydrophobic surfaces, J. Fluid Mech. 661, 402 (2010).

[27] K. Kamrin, M. Z. Bazant, and H. A. Stone, Effective slip boundary conditions for arbitrary periodic surfaces: The surface mobility tensor, J. Fluid Mech. 658, 409 (2010).

[28] D. Crowdy, Slip length for longitudinal shear flow over a dilute periodic mattress of protruding bubbles, Phys. Fluids 22, 121703 (2010).

[29] M. A. Samaha, H. Vahedi Tafreshi, and M. Gad-el Hak, Modeling drag reduction and meniscus stability of superhydrophobic surfaces comprised of random roughness, Phys. Fluids 23, 012001 (2011).

[30] C.-O. Ng and C. Y. Wang, Effective slip for stokes flow over a surface patterned with two- or threedimensional protrusions, Fluid Dyn. Res. 43, 065504 (2011).

[31] E. S. Asmolov and O. I. Vinogradova, Effective slip boundary conditions for arbitrary one-dimensional surfaces, J. Fluid Mech. 706, 108 (2012).

[32] R. Enright, M. Hodes, T. Salamon, and Y. Muzychka, Isoflux Nusselt number and slip length formulae for superhydrophobic microchannels, J. Heat Transfer 136, 012402 (2014).

[33] E. Lauga, M. P. Brenner, and H. A. Stone, Microfluidics: The no-slip boundary condition, in Handbook of Experimental Fluid Dynamics, edited by J. Foss, C. Tropea, and A. Yarin (Springer, Berlin, Heidelberg, 2005).

[34] D. Kim and W. Hwang, A template-based superhydrophobic tube structure with nanofiber forests and its mass flow characteristic, J. Micromech. Microeng. 20, 027002 (2010).

[35] R. Truesdell, A. Mammoli, P. Vorobieff, F. van Swol, and C. J. Brinker, Drag Reduction on a Patterned Superhydrophobic Surface, Phys. Rev. Lett. 97, 044504 (2006).

[36] G. McHale, N. J. Shirtcliffe, C. R. Evans, and M. I. Newton, Terminal velocity and drag reduction measurements on superhydrophobic spheres, Appl. Phys. Lett. 94, 064104 (2009).

[37] N. J. Shirtcliffe, G. McHale, M. I. Newton, and Y. Zhang, Superhydrophobic copper tubes with possible flow enhancement and drag reduction, ACS Appl. Mater. Interfaces 1, 1316 (2009).

[38] J. P. Rothstein, Slip on superhydrophobic surfaces, Annu. Rev. Fluid Mech. 42, 89 (2010).

[39] C. Lee and C.-J. Kim, Underwater Restoration and Retention of Gases on Superhydrophobic Surfaces for Drag Reduction, Phys. Rev. Lett. 106, 014502 (2011).

[40] Z. Ming, L. Jian, W. Chunxia, Z. Xiaokang, and C. Lan, Fluid drag reduction on superhydrophobic surfaces coated with carbon nanotube forests (cnts), Soft Matter 7, 4391 (2011).

[41] D. Song, R. J. Daniello, and J. P. Rothstein, Drag reduction using superhydrophobic sanded teflon surfaces, Exp. Fluids 55, 1783 (2014).

[42] M. N. Kavalenka, F. Vüllers, S. Lischker, C. Zeiger, A. Hopf, M. Röhrig, B. E. Rapp, M. Worgull, and H. Hölscher, Bioinspired air-retaining nanofur for drag reduction, ACS Appl. Mater. Interfaces 7, 10651 (2015).

[43] C. Lee and C.-J. C. Kim, Maximizing the giant liquid slip on superhydrophobic microstructures by nanostructuring their sidewalls, Langmuir 25, 12812 (2009).

[44] C. Lee, C.-H. Choi, and C.-J. Kim, Superhydrophobic drag reduction in laminar flows: A critical review, Exp. Fluids 57, 176 (2016). 
[45] P. Tsai, A. M. Peters, C. Pirat, M. Wessling, R. G. H. Lammertink, and D. Lohse, Quantifying effective slip length over micropatterned hydrophobic surfaces, Phys. Fluids 21, 112002 (2009).

[46] G. Bolognesi, C. Cottin-Bizonne, and C. Pirat, Evidence of slippage breakdown for a superhydrophobic microchannel, Phys. Fluids 26, 082004 (2014).

[47] D. Schäffel, K. Koynov, D. Vollmer, H.-J. Butt, and C. Schönecker, Local Flow Field and Slip Length of Superhydrophobic Surfaces, Phys. Rev. Lett. 116, 134501 (2016).

[48] Y. Ichikawa, K. Yamamoto, M. Yamamoto, and M. Motosuke, Near-hydrophobic-surface flow measurement by micro-3D PTV for evaluation of drag reduction, Phys. Fluids 29, 092005 (2017).

[49] P. Joseph and P. Tabeling, Direct measurement of the apparent slip length, Phys. Rev. E 71, 035303(R) (2005).

[50] P. Joseph, C. Cottin-Bizonne, J.-M. Benoît, C. Ybert, C. Journet, P. Tabeling, and L. Bocquet, Slippage of Water Past Superhydrophobic Carbon Nanotube Forests in Microchannels, Phys. Rev. Lett. 97, 156104 (2006).

[51] C. Schönecker, T. Baier, and S. Hardt, Influence of the enclosed fluid on the flow over a microstructured surface in the Cassie state, J. Fluid Mech. 740, 168 (2014).

[52] H.-J. Butt, D. Vollmer, and P. Papadopoulos, Super liquid-repellent layers: The smaller the better, Adv. Colloid Interface Sci. 222, 104 (2015).

[53] F. Vüllers, S. Peppou-Chapman, M. N. Kavalenka, H. Hölscher, and C. Neto, Effect of repeated immersions and contamination on plastron stability in superhydrophobic surfaces, Phys. Fluids 31, 012102 (2019).

[54] C.-H. Choi and C.-J. Kim, Large Slip of Aqueous Liquid Flow Over a Nanoengineered Superhydrophobic Surface, Phys. Rev. Lett. 96, 066001 (2006).

[55] L. R. J. Scarratt, B. S. Hoatson, E. S. Wood, B. S. Hawkett, and C. Neto, Durable superhydrophobic surfaces via spontaneous wrinkling of teflon af, ACS Appl. Mater. Interfaces 8, 6743 (2016).

[56] S. A. Mäkiharju, M. Perlin, and S. L. Ceccio, On the energy economics of air lubrication drag reduction, Int. J. Nav. Archit. Ocean Eng. 4, 412 (2012).

[57] H. Ling, S. Srinivasan, K. Golovin, G. McKinley, A. Tuteja, and J. Katz, High-resolution velocity measurement in the inner part of turbulent boundary layers over super-hydrophobic surfaces, J. Fluid Mech. 801, 670 (2016).

[58] H. Ling, J. Katz, M. Fu, and M. Hultmark, Effect of Reynolds number and saturation level on gas diffusion in and out of a superhydrophobic surface, Phys. Rev. Fluids 2, 124005 (2017).

[59] Fluorescence Correlation Spectroscopy, edited by R. Rigler and E. Elson (Springer, Berlin, Heidelberg, New York, 2001).

[60] S. T. Hess, S. Huang, A. A. Heikal, and W. W. Webb, Biological and chemical applications of fluorescence correlation spectroscopy: A review, Biochemistry 41, 697 (2002).

[61] K. Koynov and H.-J. Butt, Fluorescence correlation spectroscopy in colloid and interface science, Curr. Opin. Colloid Interface Sci. 17, 377 (2012).

[62] D. Wöll, Fluorescence correlation spectroscopy in polymer science, RSC Adv. 4, 2447 (2014).

[63] J. Zhang and S. Seeger, Superoleophobic coatings with ultralow sliding angles based on silicone nanofilaments, Angew. Chem. Int. Ed. 50, 6652 (2011).

[64] O. I. Vinogradova, K. Koynov, A. Best, and F. Feuillebois, Direct Measurements of Hydrophobic Slippage Using Double-Focus Fluorescence Cross-Correlation, Phys. Rev. Lett. 102, 118302 (2009).

[65] M. Gösch, H. Blom, J. Holm, T. Heino, and R. Rigler, Hydrodynamic flow profiling in microchannel structures by single molecule fluorescence correlation spectroscopy, Anal. Chem. 72, 3260 (2000).

[66] D. Magde, W. W. Webb, and E. L. Elson, Fluorescence correlation spectroscopy. iii. uniform translation and laminar flow, Biopolymers 17, 361 (1978).

[67] C. Schönecker and S. Hardt, Assessment of drag reduction at slippery, topographically structured surfaces, Microfluid. Nanofluid. 19, 199 (2015).

[68] G. Daschiel, M. Perić, J. Jovanović, and A. Delgado, The holy grail of microfluidics: Sub-laminar drag by layout of periodically embedded microgrooves, Microfluid. Nanofluid. 15, 675 (2013).

[69] E. L. Elson and D. Magde, Fluorescence correlation spectroscopy. I. Conceptual basis and theory, Biopolymers 13, 1 (1974). 